\title{
Coherence and normalisation-by-evaluation for bicategorical cartesian closed structure
}

\author{
Marcelo Fiore \\ Department of Computer Science and Technology \\ University of Cambridge \\ Cambridge, UK \\ marcelo.fiore@cl.cam.ac.uk
}

\author{
Philip Saville \\ School of Informatics \\ University of Edinburgh \\ Edinburgh, UK \\ philip.saville@ed.ac.uk
}

\begin{abstract}
We present two proofs of coherence for cartesian closed bicategories. Precisely, we show that in the free cartesian closed bicategory on a set of objects there is at most one structural 2 -cell between any parallel pair of 1-cells. We thereby reduce the difficulty of constructing structure in arbitrary cartesian closed bicategories to the level of 1-dimensional category theory. Our first proof follows a traditional approach using the Yoneda lemma. For the second proof, we adapt Fiore's categorical analysis of normalisation-by-evaluation for the simply-typed lambda calculus. Modulo the construction of suitable bicategorical structures, the argument is not significantly more complex than its 1-categorical counterpart. It also opens the way for further proofs of coherence using (adaptations of) tools from categorical semantics.
\end{abstract}

\section{CCS Concepts: • Theory of computation $\rightarrow$ Categorical semantics; Type theory.}

Keywords: bicategories, cartesian closure, coherence, type theory, normalisation, normalisation-by-evaluation

\section{ACM Reference Format:}

Marcelo Fiore and Philip Saville. 2020. Coherence and normalisationby-evaluation for bicategorical cartesian closed structure. In Proceedings of the 35th Annual ACM/IEEE Symposium on Logic in Computer Science (LICS '20), July 8-11, 2020, Saarbrücken, Germany. ACM, New York, NY, USA, 15 pages. https://doi.org/10.1145/3373718. 3394769

\section{Introduction}

Consider a pair of spans $(A \leftarrow X \rightarrow B)$ and $(B \leftarrow Y \rightarrow C)$ in a category with finite limits. By analogy with the category of

Permission to make digital or hard copies of all or part of this work for personal or classroom use is granted without fee provided that copies are not made or distributed for profit or commercial advantage and that copies bear this notice and the full citation on the first page. Copyrights for components of this work owned by others than the author(s) must be honored. Abstracting with credit is permitted. To copy otherwise, or republish, to post on servers or to redistribute to lists, requires prior specific permission and/or a fee. Request permissions from permissions@acm.org. LICS '20, July 8-11, 2020, Saarbrücken, Germany

(C) 2020 Copyright held by the owner/author(s). Publication rights licensed to ACM.

ACM ISBN 978-1-4503-7104-9/20/07...\$15.00

https://doi.org/10.1145/3373718.3394769 sets and functions, these could be thought of as relations (or tables) $A \rightsquigarrow B$ and $B \rightsquigarrow C$. How should the composite (or join) $A \rightsquigarrow C$ be defined? A natural construction is to take the pullback of the cospan $(X \rightarrow B \leftarrow Y)$ and use the associated projection maps. Because limits are unique only up to (unique) isomorphism, this definition does not satisfy the categorical unit and associativity laws. However, such laws do hold up to specified isomorphisms which satisfy coherence axioms. The resulting structure is a bicategory.

Bicategories appear widely in mathematics and theoretical computer science, arising for instance in algebra [11, 72], semantics of computation $[17,40,58]$, datatype models [1, $21]$, categorical logic [27, 37], and categorical algebra [28, $29,36]$. They also provide a natural setting for the study of many categorical concepts (e.g. [70]).

Calculating in bicategories is significantly more onerous than in either a category or a 2-category. Consider, for instance, the bicategorical version of a (co)monoid, called a pseudo-(co)monoid [23]. To give a pseudo-monoid on an object $M$ in a bicategory with finite products $(\times, 1)$ one must provide 1-cells $(1 \rightarrow M \leftarrow M \times M)$ together with invertible 2 -cells witnessing the usual categorical monoid laws. These must in turn satisfy coherence axioms corresponding to the triangle and pentagon axioms of a monoidal category. (Indeed, a pseudo-monoid in the 2-category Cat-which has products given by the usual product of categories-is exactly a monoidal category.) As in the categorical setting, every object $M$ in a bicategory with finite products has a canonical pseudo-comonoid structure $(1 \stackrel{!}{\leftarrow} M \stackrel{\Delta}{\rightarrow} M \times M)$. One may construct the witnessing 2 -cells by proving the corresponding 1-categorical result and observing that each equality corresponds to a composite of 2-cells. However, the difficulty comes with checking the coherence axioms. Because the witnessing 2-cells are composites defined using universal properties, these checks entail a series of long diagram chases. So not only is there more to check but, further, the checks themselves are a significant undertaking.

\subsection{Coherence}

One way to reduce the burden of checking coherence axioms is to prove a coherence theorem. The most famous example is Mac Lane's coherence theorem for monoidal categories, together with its pithy slogan all diagrams commute [54, 55]. 
Other notable examples include the coherence theorems for symmetric monoidal categories [47], indexed categories [56], and tricategories (weak 3-categories) [41, 43].

The case of monoidal categories might lead one to suggest that coherence is a simple matter: one 'only' needs to prove the commutativity of every diagram constructed using just structural data. Even at the level of symmetric monoidal categories, however, this is not the case, and one must take care to classify exactly which class of diagrams is coherent (see e.g. [55, Chapter XI]).

It is also worth remarking that the word 'coherence' is used in more than one way. For what might be called Mac Lanestyle coherence, one isolates a class of diagrams and proves that every diagram of this class commutes. Syntactic proofs of coherence generally make this explicit (e.g. [55]). For coherence-by-strictification, on the other hand, one shows that every instance of the weak structure is equivalent to a strict version: for example, that every bicategory is biequivalent to a 2-category. In all cases that we know of, coherenceby-strictification entails Mac Lane-style coherence. However, the proof requires a non-trivial argument (see e.g. [51, §2.4] or Section 3 below).

In this paper we provide two proofs of a Mac Lane-style coherence theorem for cartesian closed bicategories; that is, for bicategories equipped with bicategorical products and exponentials, which may be thought of as cartesian closed categories 'up to isomorphism'. Examples include the bicategories of generalised species [27] and cartesian distributors [29], bicategories of concurrent games [60], and bicategories of operads [37]. Precisely, we show the following result.

Theorem 1.1. Let $\mathbb{B}$ be any set and $\sigma, \tau: t \Rightarrow t^{\prime}$ be a parallel pair of 2-cells in the free cartesian closed bicategory on $\mathbb{B}$. Then $\sigma=\tau$.

In other words, in the free cartesian closed bicategory on a set, any two pasting diagrams with the same domain and codomain are equal. This settles a conjecture put forward by Ouaknine [59]. In the context of our [32] it establishes that, modulo the equational theory of cartesian closed bicategories, there is at most one rewrite between any two terms in the type theory $\Lambda_{\mathrm{ps}}^{\times, \rightarrow}$ for cartesian closed bicategories on a set of base types.

\subsection{Coherence as normalisation}

Our first proof of Theorem 1.1 (Section 3) follows the pattern for such results laid out by [41, 43, 47, 63]. Our second proof, by contrast, is novel. Using the 'internal language' $\Lambda_{\mathrm{ps}}^{\times, \rightarrow}$ for cartesian closed bicategories presented in [32], we reduce the problem of coherence to a normalisation problem for $\Lambda_{\mathrm{ps}}^{\times, \rightarrow}$, which we solve using semantic methods.

This 'denotational semantics' approach to coherence, which we outline in Section 4, is guided by two principles. First, to leverage the close connection between $\Lambda_{\mathrm{ps}}^{\times, \rightarrow}$ and the simplytyped lambda calculus (STLC), see Proposition 4.3. The reduction behaviour of the STLC is well-understood (see e.g. [39]) and we wish to make use of this. Second, to work with abstract properties so far as possible. This allows us to translate smoothly between categorical arguments and their bicategorical counterparts, and so provide a proof for which each step is guided by categorical understanding.

Our proof uses a version of the normalisation-by-evaluation (NbE) technique. First introduced by Berger and Schwichtenberg [12] for the STLC, normalisation-by-evaluation has become a standard tool for tackling normalisation problems and has been extended to a number of richer calculi (see e.g. [2, 5, 7, 8, 69]). Following our second principle, we proceed in the vein of categorical reconstructions of $\operatorname{NbE}$ (e.g. [6, 18, 20, 24]). Specifically, our argument closely follows that of [24], which is particularly amenable to bicategorical generalisation.

\subsection{Bicategorical structure via categorical proofs}

As Theorem 1.1 establishes the uniqueness of structural 2-cells in any cartesian closed bicategory, one obtains the following loosely-stated principle as a corollary.

To show that a pseudo structure may be constructed in any cartesian closed bicategory, it suffices to show that its categorical counterpart-that is, the version in which one only considers the 1-cells-may be constructed in any cartesian closed category (equivalently, in the STLC).

Indeed, consider a structure definable in any cartesian closed category. Such a definition is witnessed by an STLC term. We shall show in Section 4.1.1 that this STLC term corresponds to a 1-cell in a free cartesian closed bicategory and, moreover, that the $\beta \eta$-equalities it satisfies correspond to invertible 2 -cells. Theorem 1.1 then entails that any coherence axioms on these 2-cells must hold. It follows that, when it comes to constructing structure in an arbitrary cartesian closed bicategory, one may work completely 1-categorically.

This principle applies to an aforementioned example: to show that every object in a bicategory with finite products has a canonical pseudo-comonoid structure it suffices to invoke the corresponding fact in cartesian categories. For an example in the cartesian closed setting, one may consider a typed version of Statman's BAD-algebras [68]. For any object $D$ in a cartesian closed category, set $B_{1}$ to be the endo-exponential $[D \Rightarrow D]$ and let $B_{n+1}:=\left[B_{n} \Rightarrow B_{n}\right]$ (BAD-algebras arise in the context of reflexive objects $D \cong$ $[D \Rightarrow D])$. Then $B_{1}$ has a canonical monoid structure and, inductively, each $B_{n}$ has $n$ monoid structures related by distributive laws. In particular, $B_{2}$ is an nsr-object in the sense of [31]. Attempting to reconstruct this bicategorically, without coherence, is a serious undertaking; with Theorem 1.1 in hand it becomes an exercise in categorical or type-theoretic reasoning. 


\subsection{Contributions and further work}

We have three main contributions, each of which suggests further avenues for exploration.

First, Theorem 1.1 drastically reduces the difficulty of constructing structure in an arbitrary cartesian closed bicategory. This will have applications in the development of a theory of opetopic structures [26] in the cartesian closed bicategories of generalised species [27] and cartesian distributors [29], as well as in the study of the equational theory of rewriting in the STLC [32, 33].

Second, the NbE strategy is of interest in its own right. Unlike other coherence proofs, which are generally wholly syntactic (e.g. [35, 46, 74]) or wholly semantic (e.g. [63, 64]), ours combines syntax-providing a concrete handle on the problem-with the powerful tools of categorical semantics. It is therefore a middle way between the intricacies of rewriting theory or proof theory and the abstract sophistication of two-dimensional category theory. Indeed, once the expected bicategorical universal properties have been established, the argument is no more difficult than its categorical counterpart. Moreover, the wide range of categorical treatments of $\mathrm{NbE}$ suggests the approach may be applicable to other coherence problems. Possible examples include bicategories with finite coproducts (corresponding to type-theoretic sum types) or locally cartesian closed bicategories (corresponding to typetheoretic dependent sum and product types).

Finally, we build the framework for future applications. The bicategorical glueing construction developed for this proof has already been put to use in the study of the equational theory of rewriting [33]. More broadly, this paper initiates the study of the 'bicategorical semantics' of simple type theories, and of the application of categorical normalisation arguments to coherence. We see this as a stepping stone in the study of the connections between higher categories, type theories, coherence, and normalisation.

\section{Cartesian closed bicategories}

We recall the basic theory of bicategories, including the definition of cartesian closure. A summary of the key definitions is in [51]; for a more extensive introduction see e.g. [11, 13].

\subsection{Bicategories}

Bicategories axiomatise categorical structures in which the associativity and unit laws of composition hold up to coherent isomorphism.

Definition 2.1 ([11]). A bicategory $\mathcal{B}$ consists of

- A class of objects $o b(\mathcal{B})$,

- For every $X, Y \in o b(\mathcal{B})$ a hom-category $(\mathcal{B}(X, Y), \bullet, \mathrm{id})$ with objects 1-cells $f: X \rightarrow Y$ and morphisms 2-cells $\alpha: f \Rightarrow f^{\prime}: X \rightarrow Y$; composition of 2-cells is called vertical composition,

- For every $X, Y, Z \in o b(\mathcal{B})$ an identity functor $\operatorname{Id}_{X}$ : $\mathbb{1} \rightarrow \mathcal{B}(X, X)$ (for $\mathbb{1}=\{*\}$ the terminal category) and a horizontal composition functor

$$
{ }^{\circ}, Y, Z: \mathcal{B}(Y, Z) \times \mathcal{B}(X, Y) \rightarrow \mathcal{B}(X, Z),
$$

- Structural isomorphisms

$$
\begin{aligned}
\mathrm{a}_{h, g, f}:(h \circ g) \circ f & \Rightarrow h \circ(g \circ f): W \rightarrow Z \\
\mathrm{l}_{f}: \operatorname{Id}_{X} \circ f & \Rightarrow f: W \rightarrow X \\
\mathrm{r}_{g}: g \circ \operatorname{Id}_{X} & \Rightarrow g: X \rightarrow Y
\end{aligned}
$$

for every $f: W \rightarrow X, g: X \rightarrow Y$ and $h: Y \rightarrow Z$, natural in each of their arguments and satisfying axioms analogous to those for monoidal categories (note that we identify $\operatorname{Id}_{X}$ with $\left.\operatorname{Id}_{X}(*)\right)$.

When $\alpha: f \Rightarrow f^{\prime}: X \rightarrow Y$ and $\beta: g \Rightarrow g^{\prime}: Y \rightarrow Z$, one writes $g \circ \alpha$ and $\beta \circ f$ for $\operatorname{id}_{g} \circ \alpha$ and $\beta \circ \mathrm{id}_{f}$, respectively (the so-called whiskering operations).

Example 2.2. $\quad$ 1. Every bicategory $\mathcal{B}$ has an opposite bicategory $\mathcal{B}^{\mathrm{op}}$ in which just the 1-cells are reversed: one defines $\mathcal{B}^{\text {op }}(X, Y):=\mathcal{B}(Y, X)$ for all $X, Y \in o b(\mathcal{B})$.

2. Every 2-category is a bicategory in which the structural isomorphisms are all the identity.

3. For any category $\mathbb{C}$ with pullbacks there exists a $b i$ category of spans over $\mathbb{C}[11]$. The objects are those of $\mathbb{C}$, 1-cells $A \rightsquigarrow B$ are spans $A \leftarrow X \rightarrow B$, and 2-cells $(A \leftarrow X \rightarrow B) \Rightarrow\left(A \leftarrow X^{\prime} \rightarrow B\right)$ are morphisms $X \rightarrow X^{\prime}$ making the expected diagram commute. Composition is defined using pullback.

4. The bicategory Prof of profunctors (or distributors [11]) has objects categories. The hom-category $\operatorname{Prof}(\mathbb{A}, \mathbb{B})$ is the functor category $\operatorname{Fun}\left(\mathbb{B}^{o p} \times \mathbb{A}\right.$, Set $)$. The identity on $\mathbb{A}$ is the hom-functor $\mathbb{A}(-,=)$ and composition is defined using left Kan extension (see e.g. [13]).

Morphisms of bicategories are called pseudofunctors (or homomorphisms) [11]. These are mappings on objects, 1-cells and 2-cells that preserve horizontal composition up to isomorphism. Vertical composition is preserved strictly.

Definition 2.3. A pseudofunctor $\left(F, \phi^{F}, \psi^{F}\right): \mathcal{B} \rightarrow C$ between bicategories $\mathcal{B}$ and $C$ consists of

- A mapping $F_{0}: o b(\mathcal{B}) \rightarrow o b(C)$,

- A functor $F_{X, Y}: \mathcal{B}(X, Y) \rightarrow \mathcal{C}\left(F_{0} X, F_{0} Y\right)$ for every $X, Y \in o b(\mathcal{B})$ (we drop the subscripts when they are clear from context),

- An invertible 2-cell $\psi_{X}^{F}: \operatorname{Id}_{F X} \Rightarrow F\left(\operatorname{Id}_{X}\right)$ for every $X \in o b(\mathcal{B})$,

- An invertible 2-cell $\phi_{f, g}^{F}: F(f) \circ F(g) \Rightarrow F(f \circ g)$ for every $f: Y \rightarrow Z$ and $g: X \rightarrow Y$, natural in $f$ and $g$,

subject to three coherence laws. A pseudofunctor $F$ for which $\psi^{F}$ and $\phi^{F}$ are both the identity is called strict; when $\mathcal{B}$ and $C$ are 2-categories, a strict pseudofunctor $\mathcal{B} \rightarrow C$ is precisely a 2-functor.

For a property $P$, a bicategory $\mathcal{B}$ (resp. pseudofunctor $F$ ) is called locally $P$ whenever $P$ holds for each category $\mathcal{B}(X, Y)$ 
(resp. functor $F_{X, Y}$ ). In particular, a bicategory $\mathcal{B}$ is called locally small if each $\mathcal{B}(X, Y)$ is small.

Notation 2.1. We write Cat for the 2-category of small categories.

Example 2.4. 1. A monoidal category is equivalently a one-object bicategory; a monoidal functor is equivalently a pseudofunctor between one-object bicategories.

2. For a locally small bicategory $\mathcal{B}$ and $X \in o b(\mathcal{B})$ there exists the representable pseudofunctor $\mathrm{YX}: \mathcal{B}^{\mathrm{op}} \rightarrow$ Cat defined by $\mathrm{Y} X:=\mathcal{B}(-, X)$. The 2-cells $\phi^{\mathrm{Y}}$ and $\psi^{\mathrm{Y}}$ are structural isomorphisms.

For every pair of bicategories $\mathcal{B}$ and $C$ there exists a bicategory $\operatorname{Hom}(\mathcal{B}, C)$ of pseudofunctors, pseudonatural transformations and modifications (see e.g. [51]). One may think of a pseudonatural transformation $(\mathrm{k}, \overline{\mathrm{k}}): F \Rightarrow G$ as a family of 1-cells $\mathrm{k}_{X}: F X \rightarrow G X$ together with coherent isomorphisms $\overline{\mathrm{k}}_{f}: \mathrm{k}_{Y} \circ F f \Rightarrow G f \circ \mathrm{k}_{X}$ witnessing naturality. If $C$ is a 2-category, so is $\operatorname{Hom}(\mathcal{B}, C)$. In particular, for every bicategory $\mathcal{B}$ there exists a 2-category $\operatorname{Hom}\left(\mathcal{B}^{\text {op }}\right.$, Cat $)$, which one may think of as a bicategorical version of the presheaf construction.

Notation 2.2. To avoid size issues we adopt the convention that whenever we write $\operatorname{Hom}(\mathcal{B}$, Cat $)$ the bicategory $\mathcal{B}$ is small.

The appropriate notion of equivalence between bicategories is biequivalence. A biequivalence $\mathcal{B} \simeq \mathcal{C}$ consists of a pair of pseudofunctors $F: \mathcal{B} \leftrightarrows C: G$ together with equivalences $F G \simeq \operatorname{id}_{C}$ and $G F \simeq \operatorname{id}_{\mathcal{B}}$ in $\operatorname{Hom}(C, C)$ and $\operatorname{Hom}(\mathcal{B}, \mathcal{B})$ respectively. (Equivalences in an arbitrary bicategory are defined by analogy with equivalences of categories, see e.g. [52, p. 28].) Every biequivalence is locally an equivalence, hence locally fully-faithful.

\subsection{Cartesian closed bicategories}

A cartesian closed bicategory is a bicategory equipped with finite products defined as bicategorical limits (bilimits [71]), and exponentials defined as a bicategorical right adjoint ( $b i^{-}$ adjoint [42]) to every pseudofunctor $(-) \times A$. We state the definitions as biuniversal arrows [34, Chapter 9] (c.f. [27, 32]).

To avoid confusion with the 'cartesian bicategories' of Carboni and Walters $[14,16]$ we call a bicategory with finite products an $f p$-bicategory.

Definition 2.5. An $f p$-bicategory $\left(\mathcal{B}, \Pi_{n}(-)\right)$ is a bicategory $\mathcal{B}$ equipped with the following data for $A_{1}, \ldots, A_{n} \in o b(\mathcal{B})$ $(n \in \mathbb{N})$ and $k=1, \ldots, n$ :

1. A chosen object $\prod_{n}\left(A_{1}, \ldots, A_{n}\right)$,

2. Chosen projections $\pi_{k}: \prod_{n}\left(A_{1}, \ldots, A_{n}\right) \rightarrow A_{k}$,
3. For every $X \in o b(\mathcal{B})$ an adjoint equivalence

$$
\mathcal{B}\left(X, \prod_{n}(A_{1}, \ldots, \overbrace{\langle-, \ldots,=\rangle}^{\frac{\left(\pi_{1} \circ-, \ldots, \pi_{n} \circ-\right)}{\simeq}} \prod_{i=1}^{n} \mathcal{B}\left(X, A_{i}\right)\right.
$$

defined by a choice of universal arrows (e.g. [55]) with components $\varpi_{f_{1}, \ldots, f_{n}}^{(i)}: \pi_{i} \circ\left\langle f_{1}, \ldots, f_{n}\right\rangle \stackrel{\cong}{\Rightarrow} f_{i}$ for $i=$ $1, \ldots, n$.

We call the right adjoint $\langle-, \ldots,=\rangle$ the $n$-ary tupling.

An fp-bicategory has strict products if every equivalence (1) is an isomorphism. When the underlying bicategory is a 2-category, one recovers the 2-categorical (Cat-enriched) definition of finite products.

Remark 2.1. Throughout we shall assume that the unary product $\Pi_{1}(-)$ is the identity, i.e. that $\prod_{1}(A)=A, \pi_{1}^{A}=\operatorname{Id}_{A}$, $\langle f\rangle=f$ and $\omega_{f}=\mathrm{I}_{f}: \operatorname{Id} \circ f \Rightarrow f$.

Example 2.6. The bicategory of spans over a lextensive category [15] has finite biproducts (that is, finite bicategorical products which coincide with finite bicategorical coproducts) [49, Theorem 6.2]. Biproduct structure is defined using the coproduct structure of the underlying category (c.f. the biproduct structure of the category of relations).

Notation 2.3. We adopt standard categorical notation where possible. For instance, we write $A \times B$ for $\prod_{2}(A, B)$ and $f \times g$ (resp. $\tau \times \sigma$ ) for the pseudofunctorial action of the product on 1-cells (resp. 2-cells).

Definition 2.7. A cartesian closed bicategory or cc-bicategory is an fp-bicategory $\left(\mathcal{B}, \Pi_{n}(-)\right)$ equipped with the following data for every $A, B \in o b(\mathcal{B})$ :

1. A chosen object $(A \Rightarrow B)$,

2. A specified 1-cell eval $A, B:(A \Rightarrow B) \times A \rightarrow B$,

3. For every $X \in o b(\mathcal{B})$, an adjoint equivalence

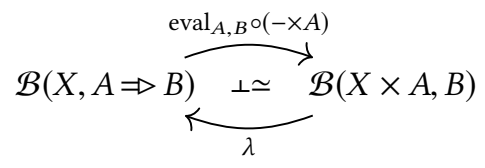

specified by a choice of universal arrows

$\varepsilon_{f}: \operatorname{eval}_{A, B} \circ(\lambda f \times A) \stackrel{\cong}{\Rightarrow} f$.

We call the functor $\lambda(-)$ currying and refer to $\lambda f$ as the currying of $f$.

A cc-bicategory is strictly cartesian closed if it has strict products and every equivalence (2) is an isomorphism. When the underlying bicategory is a 2-category, one recovers the definition of Cat-enriched cartesian closed categories, which we call 2-cc 2-categories; the prototypical example is Cat with its familiar cartesian closed structure.

Notation 2.4. As for products, we adopt standard categorical notation such as $f \Rightarrow g($ resp. $\alpha \Rightarrow \beta)$ where possible. 
Cartesian closed bicategories were first studied by Makkai [57], who introduced a cartesian closed bicategory of categories, 'anafunctors', and natural transformations. Other examples include the bicategory of generalised species [27], bicategories of operads [36], and bicategories of concurrent games [60].

By the general theory of biuniversal arrows, one may rephrase the preceding definitions in a style mirroring the 'hom-set' definition of categorical products and exponentials (see [34]). For every fp-bicategory $\left(\mathcal{B}, \Pi_{n}(-)\right)$ one obtains pseudonatural equivalences $\mathcal{B}\left(X, \prod_{i=1}^{n} A_{i}\right) \simeq \prod_{i=1}^{n} \mathcal{B}\left(X, A_{i}\right)$ (for $X, A_{1}, \ldots, A_{n} \in o b(\mathcal{B})$ and $n \in \mathbb{N}$ ) and for every ccbicategory $\left(\mathcal{B}, \Pi_{n}(-), \Rightarrow\right)$ one obtains pseudonatural equivalences $\mathcal{B}(X, A \Rightarrow B) \simeq \mathcal{B}(X \times A, B)($ for $X, A, B \in o b(\mathcal{B})$ ).

It is helpful to think of cc-bicategories as cartesian closed categories 'up to isomorphism'. Here we sketch the intuition; Proposition 4.3 makes the idea precise. To construct cc-bicategorical structure one takes the simply-typed lambda calculus (equivalently, cartesian closed structure) and replaces $\beta \eta$-equalities with invertible rewrites witnessing the reduction. For products, the $\eta$-law $f=\left\langle\pi_{1} \circ f, \ldots, \pi_{n} \circ f\right\rangle$ and $\beta$-law $\pi_{i} \circ\left\langle f_{1}, \ldots, f_{n}\right\rangle=f_{i}$ are respectively replaced by natural isomorphisms $f \stackrel{\cong}{\Rightarrow}\left\langle\pi_{1} \circ f, \ldots, \pi_{n} \circ f\right\rangle$ and $\pi_{i} \circ\left\langle f_{1}, \ldots, f_{n}\right\rangle \stackrel{\cong}{\Rightarrow} f_{i}(i=1, \ldots, n)$ given by the unit and counit of the adjunction (1). The triangle laws of an adjunction then express natural equalities: for instance, if one $\eta$-expands then $\beta$-reduces, the composite rewrite is the identity. A similar story holds for exponentials.

It is well-known that any presheaf category is cartesian closed (see e.g. [9]). This fact lifts to bicategories.

Lemma 2.8 ([66, Chapter 6]). For any small bicategory $\mathcal{B}$ the 2-category $\operatorname{Hom}\left(\mathcal{B}^{\text {op }}\right.$, Cat) has all bilimits, given pointwise, and admits a cartesian closed structure with exponentials $[P, Q](-):=\operatorname{Hom}\left(\mathcal{B}^{\text {op }}\right.$, Cat $)(\mathrm{Y}(-) \times P, Q)$.

\subsection{Cartesian closed pseudofunctors}

A cartesian closed functor preserves products and exponentials up to isomorphism. Its bicategorical counterpart preserves products and exponentials up to equivalence.

Notation 2.5. We write $A_{\bullet}$ for a finite sequence $A_{1}, \ldots, A_{n}$ $(n \in \mathbb{N})$.

Definition 2.9. An $f p$-pseudofunctor $\left(F, q^{\times}\right):\left(\mathcal{B}, \Pi_{n}(-)\right) \rightarrow$ $\left(C, \Pi_{n}(-)\right)$ is a pseudofunctor $F: \mathcal{B} \rightarrow C$ equipped with specified equivalences

$$
\left\langle F \pi_{1}, \ldots, F \pi_{n}\right\rangle: F\left(\prod_{i=1}^{n} A_{i}\right) \leftrightarrows \prod_{i=1}^{n}\left(F A_{i}\right): \mathrm{q}_{A}^{\times}
$$

for every $A_{1}, \ldots, A_{n} \in o b(\mathcal{B})(n \in \mathbb{N})$.

Definition 2.10. A cc-pseudofunctor

$$
\left(F, \mathrm{q}^{\times}, \mathrm{q}^{\Rightarrow}\right):\left(\mathcal{B}, \Pi_{n}(-), \Rightarrow\right) \rightarrow\left(C, \Pi_{n}(-), \Rightarrow\right)
$$

is an fp-pseudofunctor $\left(F, \mathrm{q}^{\times}\right)$equipped with specified equivalences $\mathrm{s}_{A, B}: F(A \Rightarrow B) \leftrightarrows(F A \Rightarrow F B): \mathrm{q}_{A, B}^{\Rightarrow}$ for every
$A, B \in o b(\mathcal{B})$, where $\mathrm{s}_{A, B}: F(A \Rightarrow B) \rightarrow(F A \Rightarrow F B)$ is the currying of $F\left(\operatorname{eval}_{A, B}\right) \circ \mathrm{q}_{A}^{\times} \Rightarrow B, A$.

Representable pseudofunctors and right biadjoints preserve all bilimits and so canonically extend to fp-pseudofunctors. As in the categorical setting, it follows from this and Lemma 2.8 that for any fp-bicategory $\left(\mathcal{B}, \Pi_{n}(-)\right)$ and $X \in o b(\mathcal{B})$ the exponential $[Y X, Q]$ in $\operatorname{Hom}\left(\mathcal{B}^{\text {op }}\right.$, Cat) may be given (up to equivalence) by $Q(-\times X)$.

\section{Coherence via the Yoneda embedding}

In this section we use the Yoneda embedding to prove coherence-by-strictification for cc-bicategories and then show how to upgrade it to a Mac Lane-style coherence result. The argument follows the tradition of such proofs for bicategories [63], monoidal categories [47], and tricategories [41, 43]. We use two ingredients. First, the fact that for any 2-category $C$ the 2-category $[C$, Cat $]$ of 2-functors, 2-natural transformations and modifications is strictly cartesian closed. Since $[C$, Cat $]$ is the Cat-enriched functor category, this follows from abstract enriched category theory (see e.g. [22, Example 5.2]). Second, a slight extension of Power's proof of coherence for bicategories with finite bilimits [63, Theorem 4.1].

Proposition 3.1. Every cartesian closed bicategory is biequivalent to a 2-cc 2-category.

Proof. Let $\left(\mathcal{B}, \Pi_{n}(-), \Rightarrow\right)$ be any cc-bicategory. By Power's result one may assume without loss of generality that $\mathcal{B}$ is a 2-category with strict products and pseudo (bicategorical) exponentials. It therefore admits a 2-categorical Yoneda embedding $\mathrm{Y}: \mathcal{B} \hookrightarrow\left[\mathcal{B}^{\text {op }}\right.$, Cat $]$. Let $\overline{\mathcal{B}}$ denote the closure of $\mathrm{Y}(o b(\mathcal{B}))$ under equivalences and factor the Yoneda embedding as $\mathcal{B} \stackrel{i}{\rightarrow} \overline{\mathcal{B}} \stackrel{j}{\rightarrow}\left[\mathcal{B}^{\text {op }}\right.$, Cat $]$, for $j$ the inclusion. $\overline{\mathcal{B}}$ is a 2-category and, by the 2-categorical Yoneda lemma, $i$ is a biequivalence.

It remains to show that $\overline{\mathcal{B}}$ admits a cartesian closed structure: we mimic Power's approach. For any $P, Q \in o b(\overline{\mathcal{B}})$ the strict exponential $(j P \Rightarrow j Q)$ exists in $\left[\mathcal{B}^{\text {op }}\right.$, Cat $]$. By definition of $\overline{\mathcal{B}}$ there exist $B, C \in o b(\mathcal{B})$ such that $P \simeq \mathrm{Y} B$ and $Q \simeq \mathrm{Y} C$. Then, $(j P \Rightarrow j Q) \simeq(\mathrm{Y} B \Rightarrow \mathrm{Y} C) \simeq \mathrm{Y}(B \Rightarrow C)$ and the exponential $(j P \Rightarrow j Q)$ is in $\overline{\mathcal{B}}$, as required.

As intimated in Section 1.1, the preceding result does not in itself entail Theorem 1.1. A further argument is required. This relies on three elements: that every biequivalence is locally faithful, and the two points of the following lemma.

Notation 3.1. For any set $\mathbb{B}$, let $\widetilde{B}$ denote the set generated by the following grammar (where $B \in \mathbb{B}$ and $n \in \mathbb{N}$ ):

$$
X_{1}, \ldots, X_{n}, X, Y::=B\left|\prod_{i=1}^{n} X_{i}\right| X \Rightarrow Y
$$

If $C$ is a cc-bicategory, every set map $\mathbb{B} \rightarrow C$ extends canonically to a map $\widetilde{\mathbb{B}} \rightarrow C$ via the cc-structure. 
Lemma 3.2. For any set $\mathbb{B}$ there exists a cc-bicategory $\mathcal{F}(\mathbb{B})$ and a mapping $\eta: \widetilde{\mathbb{B}} \rightarrow \mathcal{F}(\mathbb{B})$ such that

1. For any cc-bicategory $\left(C, \Pi_{n}(-), \Rightarrow\right)$ and set map $h$ : $\widetilde{\mathbb{B}} \rightarrow C$ canonically extending a map $\mathbb{B} \rightarrow C$ there exists a cc-pseudofunctor $h^{\#}: \mathcal{F}(\mathbb{B}) \rightarrow C$ such that $h^{\#} \circ \eta=h$.

2. If $F: \mathcal{F}(\mathbb{B}) \rightarrow C$ is a cc-pseudofunctor satisfying $F \circ \eta=$ $h$, then $F \simeq h^{\#}$.

Proof. One defines $\mathcal{F}(\mathbb{B})$ by a syntactic construction: add formal products and exponentials, and quotient by the equational theory generated by the axioms of a cc-bicategory. (Compare Mac Lane's construction of the free monoidal category [55, §VII.2]; for full details see [66, Lemma 5.2.19].)

We call $\mathcal{F}(\mathcal{B})$ the free cc-bicategory on $\mathcal{B}$. With this in hand, one may derive the Mac Lane-style form of coherence. The strategy is reminiscent of that employed by Čubrić et al. for proving normalisation of the simply-typed lambda calculus [20]. We first construct two suitable cc-pseudofunctors from the free cc-bicategory to a 2-cc 2-category. We then use their equivalence, provided by universality, to show that there is at most one 2-cell between parallel 1-cells.

First proof of Theorem 1.1. For any set of base types $\mathbb{B}$ let $H$ : $\mathcal{F}(\mathbb{B}) \rightarrow \overline{\mathcal{F}(\mathbb{B})}$ denote the biequivalence of Proposition 3.1. Every biequivalence extends canonically to a cc-pseudofunctor (see e.g. [66, Chapter 2]), so from Lemma 3.2 one obtains an equivalence $(\mathrm{k}, \overline{\mathrm{k}}): H \stackrel{\sim}{\rightarrow}\left(H_{0} \circ \eta\right)^{\#}$. This amounts to an equivalence $\mathrm{k}_{A}: H A \stackrel{\sim}{\rightarrow}\left(H_{0} \circ \eta\right)^{\#}(A)$ for every $A \in o b(\mathcal{F}(\mathbb{B}))$ and an invertible 2-cell $\overline{\mathrm{k}}_{t}: \mathrm{k}_{A} \circ H t \Rightarrow\left(H_{0} \circ \eta\right)^{\#}(t) \circ \mathrm{k}_{X}$ for every $t: X \rightarrow A$ in $\mathcal{F}(\mathbb{B})$, subject to two coherence axioms and natural in the sense that the following diagram commutes for any $\sigma: t \Rightarrow t^{\prime}$ :

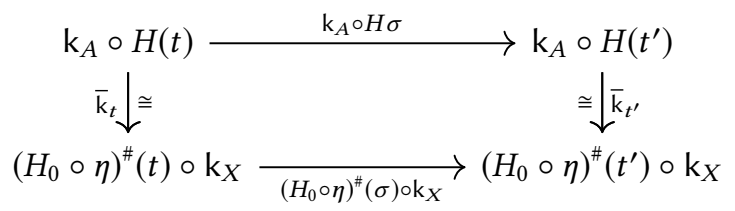

Inducting over the definition of $(-)^{\#}$, the strictness of $\overline{\mathcal{F}(\mathbb{B})}$ entails that $\left(H_{0} \circ \eta\right)^{\#}(\sigma)$ is the identity. Using this together with the fact that $\mathrm{k}_{A}$ in an equivalence, say with pseudoinverse $\mathrm{k}_{A}^{\star}$, one may extend (3) to the diagram

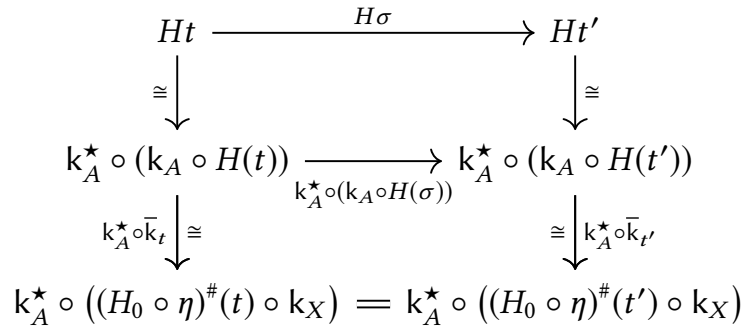

It follows that $H \sigma$ is equal to a composite that depends only on $t$ and $t^{\prime}$ (namely, the anti-clockwise route around this diagram, inverting the right-hand column) and hence that for any $\sigma, \tau: t \Rightarrow t^{\prime}$ one must have $H \sigma=H \tau$. Since $H$ is locally faithful, $\sigma=\tau$.

\section{Coherence via bicategorical $\mathrm{NbE}$}

It is well-known that the naïve strategy for proving strong normalisation of the simply-typed lambda calculus-by structural induction on terms-fails because an application $t(u)$ may contain redexes that do not occur in either $t$ or $u$. One classical solution, originally due to Tait [73], is to strengthen the inductive hypothesis using reducibility predicates. This approach was refined by Girard [38], who introduced the notion of neutral terms. These can be viewed as the obstructions to the normalisation proof: they are the terms whose formation may introduce new redexes.

$\mathrm{NbE}$ provides an alternative strategy. Loosely speaking, one constructs a model with enough intensional information to pass back and forth between syntax and semantics. One may then quote morphisms to normal terms in the syntax and unquote neutral terms to morphisms in the semantics (these operations are also known as reify and reflect). Overall, one thereby inductively defines a mapping from neutral to normal terms.

In what follows we adapt the categorical analysis of $\mathrm{NbE}$ presented in [24]. Sections 4.1-4.4 set the stage. In Section 4.1 we summarise the type theory for cc-bicategories $\Lambda_{\mathrm{ps}}^{\times,} \rightarrow$, relating it to the STLC. In Section 4.2 we recall the description of type-theoretic syntax as presheaves (c.f. [30]) and, in Section 4.3, extend it to describe syntax as Cat-valued pseudofunctors. In Section 4.4 we introduce and present the key properties of the bicategorical glueing construction. Together with the semantic interpretation, this is used in Section 4.5 to 'glue' together syntax and semantics as objects in the glueing bicategory. Finally, in Section 4.6, we complete the $\mathrm{NbE}$ argument.

The proof we are about to give is longer and more intricate than the Yoneda-embedding proof presented in Section 3, so it is worth justifying this effort.

First, one may be able to refine the $\mathrm{NbE}$ proof-for instance by considering semantic models with additional intensional information (c.f. [20]) - to extract further syntactic information, such as a normal-form result for rewrites.

Second, the $\mathrm{NbE}$ argument may be more extensible. From the perspective of theoretical computer science one would like to consider bicategorical structures as versions of categorical structures which track reductions. The structures of interest therefore include, for instance, coproducts (that is, sum types), dependent types, and notions of initial algebra (c.f. [61]). However, because these structures are not generally preserved by the Yoneda pseudofunctor, it is not clear how a Yoneda-embedding argument could be extended to cover such cases. By contrast, and as indicated in Section 1.2, the normalisation-by-evaluation strategy has been 
extended in one form or another to cover all of these structures (see e.g. $[3,5,7,10])$. This suggests that versions of the strategy we are about to present may be available where a Yoneda-embedding argument is not.

\subsection{A type theory for cartesian closed bicategories}

We shall apply our normalisation-by-evaluation argument to an 'internal language' for cartesian closed bicategories, namely the type theory $\Lambda_{\mathrm{ps}}^{\times, \rightarrow}$ of [32]. In this section we outline its key properties.

$\Lambda_{\mathrm{ps}}^{\times, \rightarrow}$ is 2-dimensional in the style of $[44,45]$ : it consists of types, terms and rewrites between terms. Thus, as well as the usual judgment $\Gamma \vdash t: A$ to indicate " $t$ is a term of type $A$ in context $\Gamma$ ", one has the judgement $\Gamma \vdash \tau: t \Rightarrow t^{\prime}: A$ to indicate " $\tau$ is a rewrite from $t$ to $t^{\prime}$, each of type $A$, in context $\Gamma$ '. Rewrites are subject to an equational theory $\equiv$ with judgements $\Gamma \vdash \sigma \equiv \tau: t \Rightarrow t^{\prime}: A$. The up-to-isomorphism nature of bicategorical composition is modelled by an explicit substitution operation, with distinguished invertible rewrites corresponding to the structural isomorphisms a, I and r. For example, for every term $(x: A \vdash t: B)$ one may explicitly substitute $x$ for $x$ to obtain the term $(x: A \vdash t\{x \mapsto x\}: B)$. These terms are not equal; they are related by the invertible rewrite $\left(x: A \vdash \iota_{t}: t \Rightarrow t\{x \mapsto x\}: B\right)$ corresponding to $\mathrm{r}$.

One obtains a syntactic cc-bicategory $\mathcal{S}(\mathbb{B})$ by mirroring the usual construction of a syntactic model for the simplytyped lambda calculus (c.f. [62]). The objects are contexts and 1-cells $\Gamma \rightarrow\left(y_{j}: B_{j}\right)_{j=1, \ldots, m}$ are $m$-tuples of terms $\left(\Gamma \vdash t_{j}: B_{j}\right)_{j=1, \ldots, m}$ modulo $\alpha$-equivalence. Similarly, 2-cells are $m$-tuples of rewrites modulo $\alpha$-equivalence and the equational theory.

4.1.1 Semantic interpretation. The semantics of $\Lambda_{\mathrm{ps}}^{\times, \rightarrow}$ extends the Lambek-style semantics for the STLC (c.f. [44, $45,67])$. For any cc-bicategory $\left(C, \Pi_{n}(-), \Rightarrow\right)$, set of base types $\mathbb{B}$ and set map $h: \widetilde{\mathbb{B}} \rightarrow C$ canonically extending a map $\mathbb{B} \rightarrow C$, there exists a semantic interpretation $h \llbracket-\rrbracket$ assigning a product $\prod_{i=1}^{n} h \llbracket A_{i} \rrbracket$ to every context $\Gamma:=\left(x_{i}: A_{i}\right)_{i=1, \ldots, n}$, a 1-cell $h \llbracket \Gamma \vdash t: A \rrbracket: \prod_{i=1}^{n} h \llbracket A_{i} \rrbracket \rightarrow h \llbracket A \rrbracket$ to every term $\Gamma \vdash t: A$, and a 2-cell $h \llbracket \Gamma \vdash \tau: t \Rightarrow t^{\prime}: A \rrbracket: h \llbracket \Gamma \vdash t: A \rrbracket \Rightarrow$ $h \llbracket \Gamma \vdash t^{\prime}: A \rrbracket$ to every rewrite $\Gamma \vdash \tau: t \Rightarrow t^{\prime}: A$. One thereby obtains a cc-pseudofunctor $h \llbracket-\rrbracket: \mathcal{S}(\mathbb{B}) \rightarrow C$ satisfying $h \llbracket-\rrbracket \circ \iota=h$, for $\iota: \widetilde{\mathbb{B}} \hookrightarrow \mathcal{S}(\mathbb{B})$ the canonical map including $\widetilde{\mathbb{B}}$ in $\mathcal{S}(\mathbb{B})$.

The semantic interpretation witnesses $\mathcal{S}(\mathbb{B})$ as the free ccbicategory on $\mathbb{B}$ in a suitably bicategorical sense. For example, $\mathcal{S}(\mathbb{B})$ is canonically biequivalent to the free cc-bicategory of Lemma 3.2, in the sense that the cc-pseudofunctors $\mathrm{J}: \mathcal{F}(\mathbb{B}) \leftrightarrows \mathcal{S}(\mathbb{B}): \mathrm{K}$ extending the respective inclusions $\widetilde{\mathbb{B}} \hookrightarrow \mathcal{S}(\mathbb{B})$ and $\widetilde{\mathbb{B}} \hookrightarrow \mathcal{F}(\mathbb{B})$ form a biequivalence. This justifies calling $\Lambda_{\mathrm{ps}}^{\times, \rightarrow}$ an 'internal language' for cc-bicategories.

The proof of Theorem 1.1 therefore reduces to proving the following theorem, in which we write $\Lambda_{\mathrm{ps}}^{\times, \rightarrow}(\mathbb{B})$ for the type theory with base types $\mathbb{B}$.
Theorem 4.1. For any set of base types $\mathbb{B}$ and any rewrites $\left(\Gamma \vdash \sigma: t \Rightarrow t^{\prime}: B\right)$ and $\left(\Gamma \vdash \tau: t \Rightarrow t^{\prime}: B\right)$ in $\Lambda_{\mathrm{ps}}^{\times, \rightarrow}(\mathbb{B})$, the judgement $\left(\Gamma \vdash \sigma \equiv \tau: t \Rightarrow t^{\prime}: B\right)$ is derivable in $\Lambda_{\mathrm{ps}}^{\times, \rightarrow}(\mathbb{B})$.

Proof of Theorem 1.1 from Theorem 4.1. The argument is similar to the proof in Section 3. If $\sigma, \tau: f \Rightarrow f^{\prime}$ are parallel 2-cells in the free cc-bicategory $\mathcal{F}(\mathbb{B})$ then, by Theorem 4.1, one has $\mathrm{J} \sigma \equiv \mathrm{J} \tau$ in $\mathcal{S}(\mathbb{B})$. Since $\mathrm{J}$ is a biequivalence, hence locally fully-faithful, it follows that $\sigma=\tau$.

To prove Theorem 4.1 we shall require the following property of $\mathcal{S}(\mathbb{B})$, which corresponds to the statement that the endofunctor canonically extending the inclusion of types into the syntactic model of the STLC is isomorphic to the identity.

Proposition 4.2. For any set of base types $\mathbb{B}$, the cc-pseudofunctor $\iota \llbracket-\rrbracket: \mathcal{S}(\mathbb{B}) \rightarrow \mathcal{S}(\mathbb{B})$ extending the inclusion $\iota:$ $\widetilde{\mathbb{B}} \hookrightarrow \mathcal{S}(\mathbb{B})$ is equivalent to the identity. Hence, $\iota \llbracket-\rrbracket$ is a biequivalence.

Proof. By Lemma 3.2, J $\simeq \iota \llbracket-\rrbracket \circ \mathrm{J}: \mathcal{F}(\mathbb{B}) \rightarrow \mathcal{S}(\mathbb{B})$. Since $\mathrm{J}$ and $\mathrm{K}$ form a biequivalence, one then has $\mathrm{id}_{\mathcal{S}(\mathbb{B})} \simeq \mathrm{J} \circ \mathrm{K} \simeq$ $(\iota \llbracket-\rrbracket \circ \mathrm{J}) \circ \mathrm{K} \simeq \iota \llbracket-\rrbracket \circ(\mathrm{J} \circ \mathrm{K}) \simeq \iota \llbracket-\rrbracket \circ \mathrm{id}_{\mathcal{S}(\mathbb{B})}$, as required.

4.1.2 $\Lambda_{\mathrm{ps}}^{\times, \rightarrow}$ as STLC 'up to isomorphism'. The rewrites of $\Lambda_{\mathrm{ps}}^{\times, \rightarrow}$ may be viewed as constructive witnesses to $\beta \eta$ equalities in the simply-typed lambda calculus, as follows. Let $\mathbb{B}$ be any set of base types, $A \in \widetilde{\mathbb{B}}$ be any type, and $\Gamma$ be any context. Write $\Lambda^{\times, \rightarrow}(\mathbb{B})$ for the set of STLC terms with base types $\mathbb{B}$, modulo $\alpha$-equivalence, and $\Lambda^{\times, \rightarrow}(\mathbb{B})(\Gamma ; A)$ for the subset of terms of type $A$ in context $\Gamma$. Similarly, write $\Lambda_{\mathrm{ps}}^{\times, \rightarrow}(\mathbb{B})$ for the set of $\Lambda_{\mathrm{ps}}^{\times, \rightarrow}(\mathbb{B})$-terms, modulo $\alpha$-equivalence, and $\Lambda_{\mathrm{ps}}^{\times, \rightarrow}(\mathbb{B})(\Gamma ; A)$ for the subset of terms of type $A$ in context $\Gamma\left(\right.$ so $\left.\Lambda_{\mathrm{ps}}^{\times, \rightarrow}(\mathbb{B})(\Gamma ; A)=o b(\mathcal{S}(\mathbb{B})(\Gamma,(x: A)))\right)$. Finally, write $\cong_{A}^{\Gamma}$ for the equivalence relation on $\Lambda_{\mathrm{ps}}^{\times, \rightarrow}(\mathbb{B})(\Gamma ; A)$ defined by $t \cong{ }_{A}^{\Gamma} t^{\prime}$ if and only if there exists a (necessarily invertible) rewrite $\tau$ such that $\Gamma \vdash \tau: t \Rightarrow t^{\prime}: A$.

Proposition 4.3. For any set of base types $\mathbb{B}$, type $A \in \widetilde{\mathbb{B}}$, and context $\Gamma$, there exist inductively-defined maps

$$
(-)_{A}^{\Gamma}: \Lambda^{\times, \rightarrow}(\mathbb{B})(\Gamma ; A) \leftrightarrows \Lambda_{\mathrm{ps}}^{\times, \rightarrow}(\mathbb{B})(\Gamma ; A):\langle-\rangle_{A}^{\Gamma}
$$

inducing a bijection

$$
\Lambda^{\times, \rightarrow}(\mathbb{B})(\Gamma ; A) /={ }_{\beta \eta} \cong \Lambda_{\mathrm{ps}}^{\times, \rightarrow}(\mathbb{B})(\Gamma ; A) / \cong_{A}^{\Gamma}
$$

between $\Lambda^{\times, \rightarrow}$-terms modulo $\beta \eta$-equality and $\Lambda_{\mathrm{ps}}^{\times,} \rightarrow$-terms modulo rewriting.

Intuitively, $(-)_{A}^{\Gamma}$ embeds STLC terms into $\Lambda_{\mathrm{ps}}^{\times, \rightarrow}$, while $\langle-\rangle_{A}^{\Gamma}$ 'evaluates' explicit substitutions into actual substitutions (c.f. [65, Definition 11]). For example, for the term $\Gamma \vdash t\{x \mapsto u\}: B$ obtained by explicitly substituting a term $\Gamma \vdash u: A$ for a variable $x$ in $t$, one recursively evaluates and performs the usual STLC capture-avoiding substitution: $\langle t\{x \mapsto u\}\rangle_{B}^{\Gamma}=\langle t\rangle_{B}^{\Gamma, x: A}\left[\langle u\rangle_{A}^{\Gamma} / x\right]$. These operations will allow us to import facts about the STLC into our bicategorical proof of coherence. 


\subsection{Syntax as presheaves}

In the tradition of algebraic type theory (e.g. [25]), the category of presheaves over an appropriate category of contexts provides a semantic universe for the study of abstract syntax [30]. In this section we recapitulate this analysis; in the next we extend it to the bicategorical setting.

4.2.1 A category of contexts. For any set of base types $\mathbb{B}$, let $\operatorname{Con}_{\widetilde{\mathbb{B}}}$ denote the category of contexts over $\widetilde{\mathbb{B}}$. This has objects contexts with $i^{\text {th }}$ variable $x_{i}$ (for some enumeration of variables $\left.\left\{x_{i}\right\}_{1 \leq i \in \mathbb{N}}\right)$ and morphisms context renamings: a morphism $r:\left(x_{i}: A_{i}\right)_{i=1, \ldots, n} \rightarrow\left(x_{j}: B_{j}\right)_{j=1, \ldots, m}$ is a map $r:\{1, \ldots, n\} \rightarrow\{1, \ldots, m\}$ such that $A_{i}=B_{r(i)}$ for all $i$. The coproduct structure is given by context concatenation and the unit is the empty context. Abstractly, $\mathrm{Con}_{\widetilde{B}}$ is (isomorphic to) the free strict cocartesian category on $\widetilde{\mathbb{B}}$.

We denote the universal embedding of $\widetilde{\mathbb{B}}$ into $\operatorname{Con}_{\widetilde{B}}$ by $[-]$; thus, [-] coerces the type $A$ into the unary context $\left(x_{1}: A\right)$, and the coproduct $\Gamma+[A]$ is the weakening of $\Gamma$ by a fresh variable of type $A$. The notation is chosen to suggest a list of length one.

4.2.2 Presheaves over $\operatorname{Con}_{\widetilde{\mathbb{B}}}$. The syntax of the simplytyped lambda calculus with base types $\mathbb{B}$ is described by families of covariant presheaves over $\operatorname{Con}_{\widetilde{\mathbb{B}}}$. For any type $A \in \widetilde{\mathbb{B}}$, the set of terms-in-context defines a presheaf $\mathrm{L}(-; A)$ by $\mathrm{L}(\Gamma ; A):=\{t \mid \Gamma \vdash t: A\} /=_{\alpha}$. The functorial action is given by renaming: for a context renaming $r$ one employs the admissibility of renaming in the STLC to define $\mathrm{L}(r ; A)(t):=$ $t\left[x_{r(i)} / x_{i}\right]$.

The Yoneda embedding $\mathbf{y}$ yields a presheaf of variables: for any type $A \in \widetilde{\mathbb{B}}$ and context $\Gamma$ one identifies $\operatorname{Con}_{\widetilde{\mathbb{B}}}(\Gamma ;[A])$ with the set of variable projections, giving a presheaf $\mathrm{V}(-; A)$ defined by $\mathrm{V}(\Gamma ; A)=\mathrm{y}([A])=\{x \mid \Gamma \vdash x: A\}$. The wellknown fact that $[\mathrm{y} A, Q] \cong Q(-\times A)$ in any presheaf category over a cartesian category corresponds to the observation that the exponential presheaf $[\mathrm{y} A, \mathrm{~L}(-; B)] \cong \mathrm{L}(-+[A] ; B)$ consists of terms of type $B$ in a context extended by a fresh variable of type $A$ (note that, since $\operatorname{Con}_{\widetilde{\mathbb{B}}}$ is strict cocartesian, its opposite category is strict cartesian).

For the purposes of $\mathrm{NbE}$, two further families of presheaves are of particular importance. These are the presheaves of neutral and (long- $\beta \eta$ ) normal terms, characterised by the families of judgements $\Gamma \vdash_{M} t: A$ and $\Gamma \vdash_{N} t: A$ (respectively) of Figure 1 (see e.g. [39, Chapter 4]). We maintain our convention of using $n$-ary products.

Since the sets of neutral and normal terms are invariant under renaming, for every type $A \in \widetilde{\mathbb{B}}$ one now has four presheaves $\mathrm{Con}_{\widetilde{\mathbb{B}}} \rightarrow$ Set, defined at $\Gamma \in \mathrm{Con}_{\widetilde{\mathbb{B}}}$ as follows:

$$
\begin{aligned}
\mathrm{L}(\Gamma ; A) & :=\{t \mid \Gamma \vdash t: A\} /={ }_{\alpha} \\
\mathrm{V}(\Gamma ; A) & :=\mathrm{y}[A]=\{x \mid \Gamma \vdash x: A\} /={ }_{\alpha} \\
\mathrm{M}(\Gamma ; A) & :=\left\{t \mid \Gamma \vdash_{M} t: A\right\} /={ }_{\alpha} \\
\mathrm{N}(\Gamma ; A) & :=\left\{t \mid \Gamma \vdash_{N} t: A\right\} /={ }_{\alpha}
\end{aligned}
$$

$$
\begin{aligned}
& \overline{x_{1}: A_{1}, \ldots, x_{n}: A_{n} \vdash_{M} x_{i}: A_{i}} \text { var } \\
& \frac{\Gamma \vdash_{M} t: \prod_{i=1}^{n} A_{i}}{\Gamma \vdash_{M} \pi_{k}(t): A_{k}} \operatorname{proj}_{k}(k=1, \ldots, n) \\
& \frac{\Gamma \vdash_{M} t: X \Rightarrow Y \quad \Gamma \vdash_{N} u: X}{\Gamma \vdash_{M} t(u): Y} \text { app } \\
& \frac{\Gamma \vdash_{N} t_{i}: A_{i} \quad(i=1, \ldots, n)}{\Gamma \vdash_{N}\left\langle t_{1}, \ldots, t_{n}\right\rangle: \prod_{i=1}^{n} A_{i}} \text { tuple } \\
& \frac{\Gamma, x: X \vdash_{N} t: Y}{\Gamma \vdash_{N} \lambda x . t: X \Rightarrow Y} \text { lam } \frac{\Gamma \vdash_{M} t: B}{\Gamma \vdash_{N} t: B} \text { inc (B a base type) }
\end{aligned}
$$

Figure 1. Neutral and normal terms in the STLC.

Each rule of Figure 1 defines a morphism on these indexed families of presheaves, as shown below. The mappings are just the operations on terms. In each case naturality follows from the definition of the meta-operation of capture-avoiding substitution, in particular the fact that substitution passes through the various constructors and respects $\alpha$-equivalence.

$$
\begin{aligned}
\operatorname{var}\left(-; A_{i}\right) & : \mathrm{V}\left(-; A_{i}\right) \Rightarrow \mathrm{M}\left(-; A_{i}\right) \\
\operatorname{proj}_{k}\left(-; A_{\bullet}\right) & : \mathrm{M}\left(-; \prod_{i=1}^{n} A_{i}\right) \Rightarrow \mathrm{M}\left(-; A_{k}\right) \quad(1 \leq k \leq n) \\
\operatorname{app}(-; A, B) & : \mathrm{M}(-; X \Rightarrow Y) \times \mathrm{N}(-; X) \Rightarrow \mathrm{M}(-; Y) \\
\operatorname{tup}\left(-; A_{\bullet}\right) & : \prod_{i=1}^{n} \mathrm{~N}\left(-; A_{i}\right) \Rightarrow \mathrm{N}\left(-; \prod_{i=1}^{n} A_{i}\right) \\
\operatorname{lam}(-; X, Y) & : \mathrm{N}(-+[X] ; Y) \Rightarrow \mathrm{N}(-; X \Rightarrow Y) \\
\operatorname{inc}(-; B) & : \mathrm{M}(-; B) \Rightarrow \mathrm{N}(-; B) \quad(B \in \mathbb{B})
\end{aligned}
$$

\subsection{Syntax as pseudofunctors}

In this section we deal with two questions. First, how does one translate the presheaves and natural transformations of (5) and (6) into bicategorical constructions? Second, how does one define neutral and normal terms in $\Lambda_{\mathrm{ps}}^{\times, \rightarrow}$ ? We shall answer these questions in a manner that allows us to leverage properties of the STLC and elements of the NbE proof in [24].

4.3.1 From categories to locally discrete bicategories. Because the contexts of $\Lambda_{\mathrm{ps}}^{\times, \rightarrow}$ are exactly the same as those of the STLC, we may describe its syntax by presheaves over the category of contexts $\operatorname{Con}_{\widetilde{B}}$. To place this within the bicategorical setting, we treat $\mathrm{Con}_{\widetilde{\mathbb{B}}}$ as a locally discrete bicategory. To help us track whether we mean a set, a set treated as a discrete category, or a set treated as a locally discrete bicategory, we introduce the following notation.

\section{Notation 4.1}

1. For a set $S$, write $\partial S$ for the discrete category with objects the elements of $S$. Similarly, write $\partial f$ for the discrete functor $\partial S \rightarrow \partial S^{\prime}$ induced by the set map $f: S \rightarrow S^{\prime}$. 
2. a. For a category $\mathbb{C}$, write $\mathrm{d} \mathbb{C}$ for the locally discrete 2-category with objects those of $\mathbb{C}$ and hom-categories $(\mathrm{d} \mathbb{C})(X, Y):=\partial(\mathbb{C}(X, Y))$.

b. Write $\mathrm{d} F$ for the locally discrete 2 -functor $\mathrm{d} \mathbb{C} \rightarrow \mathrm{d} \mathbb{D}$ induced from the functor $F: \mathbb{C} \rightarrow \mathbb{D}$ by setting $(\mathrm{d} F) X:=F X$ and $(\mathrm{d} F)_{X, Y}:=\partial\left(F_{X, Y}\right)$.

c. Write $\mathrm{d} \mu$ for the locally discrete 2-natural transformation $\mathrm{d} F \Rightarrow \mathrm{d} G$ induced from the natural transformation $\mu: F \Rightarrow G: \mathbb{C} \rightarrow \mathbb{D}$ by setting $(\mathrm{d} \mu)_{C}:=\mu_{C}$.

The $\mathrm{d}(-)$ construction will be our main technical tool for constructing (degenerate) bicategorical structure from categorical data. The next lemma collects together some of its important properties. The proofs are not especially difficult, but stating all the details precisely takes some care. We write $\operatorname{Fun}(\mathbb{C}, \mathbb{D})$ for the functor category from $\mathbb{C}$ to $\mathbb{D}$.

Lemma 4.4. Let $\mathbb{C}$ and $\mathbb{D}$ be categories. Then:

1. $(\mathrm{d} \mathbb{C})^{\mathrm{op}}=\mathrm{d}\left(\mathbb{C}^{\mathrm{op}}\right)$.

2. There exists an isomorphism of 2-categories $\mathrm{d}(\operatorname{Fun}(\mathbb{C}, \mathbb{D}))$ $\cong \operatorname{Hom}(\mathrm{d} \mathbb{C}, \mathrm{dD})$.

3. There exists an injective-on-objects, locally isomorphic 2-functor $\iota: \mathrm{d}(\operatorname{Fun}(\mathbb{C}$, Set $)) \hookrightarrow \operatorname{Hom}(\mathrm{d} \mathbb{C}$, Cat $)$, which induces a commutative diagram

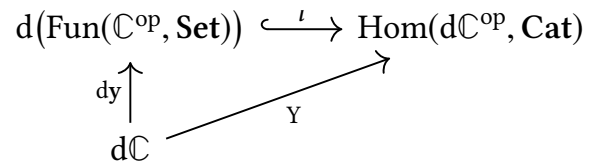

In particular, $\mathrm{Y}(C)=\mathrm{dy}(C)$ for all $C \in \mathrm{ob}(\mathbb{C})$.

4. If $\mathbb{C}$ is cartesian (resp. cartesian closed) as a 1-category, then $\mathrm{d} \mathbb{C}$ has finite products (resp. is cartesian closed) as a 2-category.

Hence, the category of contexts gives rise to a locally discrete bicategory of contexts $\mathrm{dCon}_{\widetilde{\mathbb{B}}}$, whose opposite canonically admits fp-structure. The universal property characterising $\operatorname{Con}_{\widetilde{\mathbb{B}}}$ op as the free cartesian category on $\widetilde{\mathbb{B}}$ becomes the following weak universal property among fp-bicategories: because bicategorical products are unique only up to equivalence, one cannot hope for uniqueness on the nose.

Lemma 4.5. For any set of base types $\mathbb{B}$, any cc-bicategory $\left(\mathcal{X}, \Pi_{n}(-), \Rightarrow\right)$, and any set map $s: \widetilde{\mathbb{B}} \rightarrow \mathcal{X}$ canonically extending a map $\mathbb{B} \rightarrow \mathcal{X}$, there exists an $f p$-pseudofunctor $\left(\underline{s}, \mathrm{q}^{\times}\right): \mathrm{dCon}_{\widetilde{B}}^{\text {op }} \rightarrow \mathcal{X}$ making the following commute:

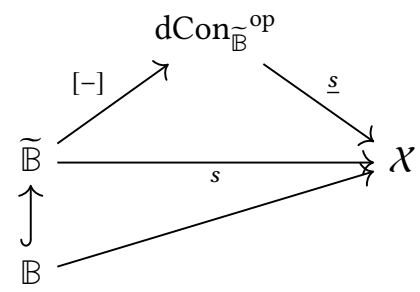

Remark 4.1. As in the 1-categorical case, $\underline{s}$ coincides with the semantic interpretation $s \llbracket-\rrbracket$ (arising from the cc-structure of $\mathcal{X}$ and the set map $s$ ) on contexts and renamings.
4.3.2 Syntax as pseudofunctors. By Lemma 4.4, every

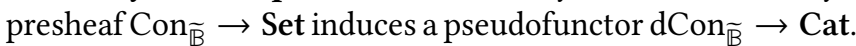
We now use Proposition 4.3 to define correlates of the presheaves (5) and natural transformations (6) for $\Lambda_{\mathrm{ps}}^{\times, \rightarrow}$. For every $A \in \widetilde{\mathbb{B}}$ one obtains four presheaves $\operatorname{Con}_{\widetilde{\mathbb{B}}} \rightarrow$ Set, defined as follows:

$$
\begin{aligned}
\mathcal{L}(\Gamma ; A) & \left.:=\{(t))_{A}^{\Gamma} \mid t \in \mathrm{L}(\Gamma ; A)\right\} \\
\mathcal{V}(\Gamma ; A) & \left.:=\{(t))_{A}^{\Gamma} \mid t \in \mathrm{V}(\Gamma ; A)\right\} \\
\mathcal{M}(\Gamma ; A): & \left.=\{(t))_{A}^{\Gamma} \mid t \in \mathrm{M}(\Gamma ; A)\right\} \\
\mathcal{N}(\Gamma ; A) & \left.:=\{(t))_{A}^{\Gamma} \mid t \in \mathrm{N}(\Gamma ; A)\right\}
\end{aligned}
$$

Where $\mathrm{K}(-; A) \in\{\mathrm{V}(-; A), \mathrm{M}(-; A), \mathrm{N}(-; A), \mathrm{L}(-; A)\}$ and $\mathcal{K}(-; A) \in\{\mathcal{V}(-; A), \mathcal{M}(-; A), \mathcal{N}(-; A), \mathcal{L}(-; A)\}$ denotes the image of $\mathrm{K}(-; A)$ under $(-)$, the functorial action on a context renaming $r: \Gamma \rightarrow \Delta$ is $\mathcal{K}(r ; A)\left((t)_{A}^{\Gamma}\right):=\left(t\left[x_{r(i)} / x_{i}\right]\right)_{A}^{\Delta}$.

This formulation has two benefits. First, it gives a reasonable notion of neutral and normal terms in $\Lambda_{\mathrm{ps}}^{\times, \rightarrow}$ without having to consider the normalisation behaviour of terms in a 2-dimensional type theory with explicit substitutions. Second, it allows us to use many of the details of [24]. For example, since the map $(-)_{A}^{\Gamma}$ is an injection for every type $A \in \widetilde{\mathbb{B}}$ and context $\Gamma$, every $(-)_{A}^{(=)}$defines a natural isomorphism $\mathrm{K}_{A} \Rightarrow \mathcal{K}_{A}$. Composing with the natural transformations of (6), one obtains the following natural transformations:

$$
\begin{aligned}
\operatorname{var}\left(-; A_{i}\right) & : \mathcal{V}\left(-; A_{i}\right) \Rightarrow \mathcal{M}\left(-; A_{i}\right) \\
\operatorname{proj}_{k}\left(-; A_{\bullet}\right) & : \mathcal{M}\left(-; \prod_{i=1}^{n} A_{i}\right) \Rightarrow \mathcal{M}\left(-; A_{k}\right)(1 \leq k \leq n) \\
\operatorname{app}(-; X, Y) & : \mathcal{M}(-; X \Rightarrow Y) \times \mathcal{N}(-; X) \Rightarrow \mathcal{M}(-; Y) \\
\operatorname{tup}\left(-; A_{\bullet}\right) & : \prod_{i=1}^{n} \mathcal{N}\left(-; A_{i}\right) \Rightarrow \mathcal{N}\left(-; \prod_{i=1}^{n} A_{i}\right) \\
\operatorname{lam}(-; X, Y) & : \mathcal{N}(-+[X] ; Y) \Rightarrow \mathcal{N}(-; X \Rightarrow Y) \\
\operatorname{inc}(-; B) & : \mathcal{M}(-; B) \Rightarrow \mathcal{N}(-; B) \quad(B \in \mathbb{B})
\end{aligned}
$$

\subsection{Bicategorical glueing}

The glueing of bicategories along a pseudofunctor is defined by analogy with the categorical construction (see e.g. [19]). Here we merely provide an outline; for details, see $[33,66]$.

\section{Definition 4.6.}

1. Let $F: \mathcal{A} \rightarrow C$ and $G: \mathcal{B} \rightarrow C$ be pseudofunctors between bicategories. The comma bicategory $(F \downarrow G)$ has objects triples $(A \in o b(\mathcal{A}), f: F A \rightarrow G B, B \in o b(\mathcal{B}))$. The 1-cells $(A, f, B) \rightarrow\left(A^{\prime}, f^{\prime}, B^{\prime}\right)$ are triples $(p, \alpha, q)$, where $p: A \rightarrow A^{\prime}$ and $q: B \rightarrow B^{\prime}$ are 1-cells and $\alpha$ is an invertible 2-cell $\alpha: f^{\prime} \circ F p \Rightarrow G q \circ f$. The 2-cells $(p, \alpha, q) \Rightarrow\left(p^{\prime}, \alpha^{\prime}, q^{\prime}\right)$ are pairs of 2-cells $\left(\sigma: p \Rightarrow p^{\prime}\right.$, $\left.\tau: q \Rightarrow q^{\prime}\right)$ satisfying the following cylinder condition:

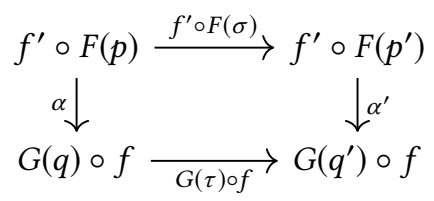


Identities and horizontal composition are given by the following pasting diagrams.
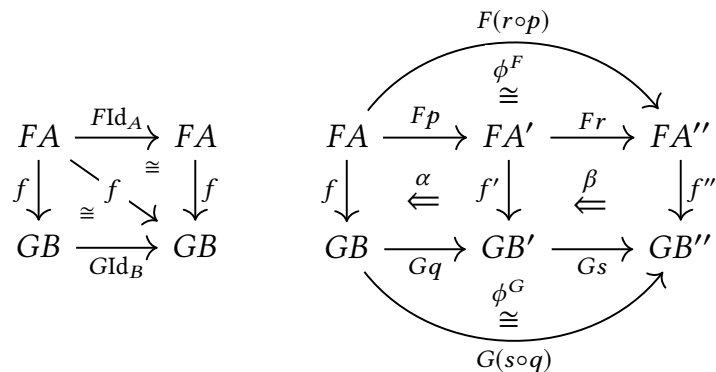

Vertical composition, the identity 2-cell, and the structural isomorphisms are given component-wise.

2. The glueing bicategory $\operatorname{gl}(\mathfrak{J})$ of bicategories $\mathcal{B}$ and $C$ along a pseudofunctor $\mathfrak{J}: \mathcal{B} \rightarrow C$ is the comma bicategory $\left(\operatorname{id}_{C} \downarrow \mathfrak{J}\right)$.

Notation 4.2. We denote the evident projection pseudofunctor by $\pi: \operatorname{gl}(\mathfrak{J}) \rightarrow \mathcal{B}$.

We adopt the terminology of [24]. For any pseudofunctor $\mathfrak{J}: \mathcal{B} \rightarrow \mathcal{X}$ one obtains the relative hom-pseudofunctor $\langle\mathfrak{J}\rangle: \mathcal{X} \rightarrow \operatorname{Hom}\left(\mathcal{B}^{\text {op }}\right.$, Cat) by setting $\langle\mathfrak{J}\rangle X:=\mathcal{X}(\mathfrak{I}(-), X)$ on objects, and likewise on 1-cells and 2-cells.

We call the glueing bicategory $\operatorname{gl}(\langle\mathfrak{J}\rangle)$ associated to a relative hom-pseudofunctor the bicategory of $\mathcal{B}$-intensional Kripke relations of arity $\mathfrak{J}$. This may be viewed as an intensional, bicategorical, version of the category of Kripke relations (c.f. [4, 24]). Indeed, an object in this bicategory consists of a pseudofunctor $P: \mathcal{B}^{\text {op }} \rightarrow$ Cat together with a chosen object $X \in o b(X)$ and a pseudonatural transformation $(\mathrm{k}, \overline{\mathrm{k}}): P \Rightarrow \mathcal{X}(\mathfrak{J}(-), X)$. In Section 4.3 we shall instantiate $P$ with syntax and $(\mathrm{k}, \overline{\mathrm{k}})$ with semantic interpretation.

The relative hom-pseudofunctor preserves all bilimits that exist in its domain and, for any pseudofunctor $\mathfrak{J}: \mathcal{B} \rightarrow \mathcal{X}$, there exists a pseudonatural transformation

$$
(l, \bar{l}): \mathrm{Y} \Rightarrow\langle\mathfrak{J}\rangle \circ \mathfrak{J}: \mathcal{B} \rightarrow \operatorname{Hom}\left(\mathcal{B}^{\text {op }} \text {, Cat }\right)
$$

given by the functorial action of $\mathfrak{J}$ on hom-categories. One may therefore define the following.

Definition 4.7. For any pseudofunctor $\mathfrak{J}: \mathcal{B} \rightarrow \mathcal{X}$, define the extended Yoneda pseudofunctor $\underline{\mathrm{Y}}: \mathcal{B} \rightarrow \operatorname{gl}(\langle\mathfrak{J}\rangle)$ by set$\operatorname{ting} \underline{\mathrm{Y}} B:=\left(\mathrm{Y} B,(l, \bar{l})_{(-, B)}, \mathfrak{J} B\right), \underline{\mathrm{Y} f}:=\left(\mathrm{Y} f,\left(\phi_{-, f}^{\mathfrak{J}}\right)^{-1}, \mathfrak{J} f\right)$, and $\underline{\mathrm{Y}}\left(\tau: f \Rightarrow f^{\prime}: B \rightarrow B^{\prime}\right):=(\mathrm{Y} \tau, \mathfrak{J} \tau)$. The cylinder condition holds by the naturality of $\phi^{\mathfrak{J}}$, and the 2-cells $\phi \underline{\underline{Y}}$ and $\psi \underline{\underline{Y}}$ are $\left(\phi^{\mathrm{Y}}, \phi^{F}\right)$ and $\left(\psi^{\mathrm{Y}}, \psi^{F}\right)$, respectively.

For cartesian closed categories $\mathbb{B}$ and $\mathbb{C}$ it is well-known that, if $F: \mathbb{B} \rightarrow \mathbb{C}$ is a product-preserving functor and $\mathbb{C}$ has pullbacks, then the glueing category $\mathrm{gl}(F)$ is cartesian closed with forgetful functor $\mathrm{gl}(F) \rightarrow \mathbb{B}$ strictly cartesian closed (e.g. [19, 50, 53]). This lifts to the bicategorical setting.

Definition 4.8 (e.g. [48]). A pullback of $\left(X_{1} \rightarrow X_{0} \leftarrow X_{2}\right)$ in a bicategory $\mathcal{B}$ is a bilimit (see e.g. [71]) for the strict pseudofunctor $X:(1 \rightarrow 0 \leftarrow 2) \rightarrow \mathcal{B}$ determined by the cospan.

Theorem $4.9([33,66])$. $\operatorname{Let}\left(\mathcal{B}, \Pi_{n}(-), \Rightarrow\right)$ and $\left(C, \Pi_{n}(-), \Rightarrow\right)$ be cc-bicategories and assume that $C$ has pullbacks. Then for any $f p$-pseudofunctor $\left(\mathfrak{J}, \mathrm{q}^{\times}\right): \mathcal{B} \rightarrow C$ the glueing bicategory gl(J) is cartesian closed with forgetful pseudofunctor $\pi: \operatorname{gl}(\mathfrak{J}) \rightarrow \mathcal{B}$ strictly cartesian closed (in the sense of [32, Definition VIII.3]).

The proof is similar to the categorical construction (e.g. [53, Proposition 2]). For a family of objects $\left(C_{i}, c_{i}, B_{i}\right)_{i=1, \ldots, n}$ the $n$-ary product $\prod_{i=1}^{n}\left(C_{i}, c_{i}, B_{i}\right)$ is the tuple

$$
\left(\prod_{i=1}^{n} C_{i}, \mathrm{q}_{B_{\bullet}}^{\times} \circ \prod_{i=1}^{n} c_{i}, \prod_{i=1}^{n} B_{i}\right)
$$

For $\underline{C}:=(C, c, B)$ and $\underline{C^{\prime}}:=\left(C^{\prime}, c^{\prime}, B^{\prime}\right)$ in $\operatorname{gl}(\mathfrak{J})$ the exponential $\underline{C} \Rightarrow C^{\prime}$ is the left-hand vertical leg of the following pullback diagram, in which $\mathrm{s}_{B, B^{\prime}}$ is the exponential transpose of $\mathfrak{I}\left(\right.$ eval $\left._{B, B^{\prime}}\right) \circ \mathrm{q}_{B \Rightarrow B^{\prime}, B}^{\times}$and the unlabelled arrow is $\lambda\left(\operatorname{eval}_{\mathfrak{J} B, \mathfrak{J} B^{\prime}} \circ\left(\left(\mathfrak{J} B \Rightarrow \mathfrak{J} B^{\prime}\right) \times c\right)\right)$ :

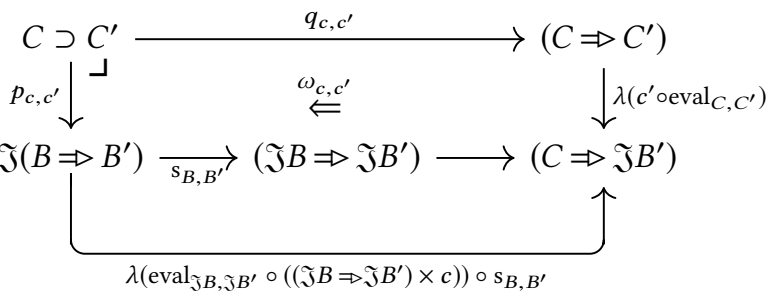

Together, Lemma 2.8 and Theorem 4.9 entail the following.

Corollary 4.10. For any cc-bicategory $\left(X, \Pi_{n}(-), \Rightarrow\right)$ and any pseudofunctor $\mathfrak{I}: \mathcal{B} \rightarrow \mathcal{X}$ the bicategory gl( $\langle\mathfrak{J}\rangle)$ of $\mathcal{B}$-intensional Kripke relations of arity $\mathfrak{J}$ admits a cartesian closed structure with forgetful pseudofunctor gl $(\langle\mathfrak{J}\rangle) \rightarrow \mathcal{X}$ strictly cartesian closed.

Just as one may explicitly describe exponentiating by a representable in a presheaf category or in the 2-category $\operatorname{Hom}\left(\mathcal{B}^{\text {op }}, \mathrm{Cat}\right)$, so one may describe exponentiating by an 'extended representable' in the glueing bicategory. We only need the result for when $\mathcal{B}$ is a 2-category.

Proposition 4.11 (c.f. [24, p. 8]). For any small 2-category $\mathcal{B}$ with bicategorical products and exponentials $\left(\Pi_{n}(-), \Rightarrow\right)$, cc-bicategory $\left(X, \Pi_{n}(-), \Rightarrow\right)$ and $f p$-pseudofunctor $\left(\mathfrak{J}, \mathrm{q}^{\times}\right)$: $\mathcal{B} \rightarrow \mathcal{X}$, the exponential $\underline{Y} B \Rightarrow(K,(\mathrm{k}, \overline{\mathrm{k}}), X)$ in $\mathrm{gl}(\langle\mathfrak{J}\rangle)$ may be given explicitly by the following composite in $\operatorname{Hom}\left(\mathcal{B}^{\text {op }}\right.$, Cat):

$$
[\mathrm{Y} B, K] \stackrel{[\mathrm{Y} B,(\mathrm{k}, \overline{\mathrm{k}})]}{\longrightarrow}[\mathrm{Y} B,\langle\mathfrak{J}\rangle X] \stackrel{u_{B, X}}{\longrightarrow}\langle\mathfrak{J}\rangle(\mathfrak{J} B \Rightarrow X)
$$

Here $u_{B, X}$ is the equivalence $[\mathrm{Y} B,\langle\mathfrak{J}\rangle X] \simeq X(\mathfrak{I}(-), \mathfrak{J} B \Rightarrow X)$ obtained by composing the equivalences arising from (1) the identification of $(\langle\mathfrak{J}\rangle X)(-\times B)$ as $[\mathrm{YB},\langle\mathfrak{J}\rangle X]$; (2) the fact that $\mathfrak{J}$ preserves products; (3) the definition of exponentials. 


\subsection{Glueing syntax and semantics}

We now 'glue' the syntactic data from Section 4.3 to the semantic interpretation of Section 4.1.1 to construct data in the bicategory of intensional Kripke relations.

Fix a cc-bicategory $\left(\mathcal{X}, \Pi_{n}(-), \Rightarrow\right)$ and consider an interpretation $\mathbb{B} \rightarrow \mathcal{X}$ of base types in $\mathcal{X}$ with canonical extension $s: \widetilde{\mathbb{B}} \rightarrow \mathcal{X}$. By Lemma 4.5 and Remark 4.1, this further extends to an fp-pseudofunctor $\underline{s}: \mathrm{dCon}_{\widetilde{\mathbb{B}}}$ op $\rightarrow X$ that coincides with the semantic interpretation $s \llbracket-\rrbracket$ on contexts. We show that the syntax and semantics of $\Lambda_{\mathrm{ps}}^{\times, \rightarrow}$ determine objects in the glueing bicategory gl $(\langle\underline{s}\rangle)$, and that the typing rules determine 1 -cells.

4.5.1 From terms to glued objects. The key observation is that the interpretation of $\Lambda_{\mathrm{ps}}^{\times, \rightarrow}$-terms is pseudonatural (Note that, since $\underline{s}$ is contravariant, the composite $\mathcal{X}(\underline{s}(-), X)$ $=\mathcal{X}(s \llbracket-\rrbracket, X)$ is covariant.)

Lemma 4.12. For every set of base types $\mathbb{B}$ and every $A \in \widetilde{\mathbb{B}}$ the semantic interpretation $s \llbracket-\rrbracket: \mathcal{S}(\mathbb{B}) \rightarrow \mathcal{X}$ forms the components of a pseudonatural transformation $(s \llbracket-\rrbracket, \overline{s \llbracket-\rrbracket})$ : $\mathrm{d} \mathcal{L}(-; A) \Rightarrow \mathcal{X}(s \llbracket-\rrbracket, s \llbracket A \rrbracket): \mathrm{dCon}_{\widetilde{\mathbb{B}}} \rightarrow$ Cat.

The preceding construction restricts to neutral and normal terms, giving pseudonatural transformations

$$
\begin{gathered}
\mathrm{d} \mathcal{M}(-; A) \stackrel{\left(s \llbracket-\rrbracket,\left.\overline{s \llbracket-\rrbracket}\right|_{M}\right.}{=} \mathcal{X}(s \llbracket-\rrbracket, s \llbracket A \rrbracket) \\
\mathrm{d} \mathcal{N}(-; A) \stackrel{\left(s \llbracket-\rrbracket,\left.\overline{s \llbracket-\rrbracket}\right|_{N}\right.}{=} \mathcal{X}(s \llbracket-\rrbracket, s \llbracket A \rrbracket)
\end{gathered}
$$

One thereby obtains the following glued objects in $\operatorname{gl}(\langle\underline{s}\rangle)$ for every type $A \in \widetilde{\mathbb{B}}$ :

$$
\begin{aligned}
\mu_{A} & :=\left(\mathrm{d} \mathcal{M}(-; A),\left.(s \llbracket-\rrbracket, \overline{s \llbracket-\rrbracket})\right|_{M}, s \llbracket A \rrbracket\right) \\
\eta_{A} & :=\left(\mathrm{d} \mathcal{N}(-; A),\left.(s \llbracket-\rrbracket, \overline{s \llbracket-\rrbracket})\right|_{N}, s \llbracket A \rrbracket\right)
\end{aligned}
$$

Finally, for variables, one takes

$$
v_{A}:=\underline{\mathrm{Y}}([A])=\left(\mathrm{d} \mathcal{V}(-; A),(l, \bar{l})_{(-, A)}, s \llbracket A \rrbracket\right)
$$

for $(l, \bar{l})_{(-, A)}$ the pseudonatural transformation of (10).

4.5.2 From rules to glued 1-cells. We now lift the natural transformations of (8)-viewed as locally discrete pseudonatural transformations-to morphisms in $\mathrm{gl}(\langle\underline{s}\rangle)$. For the lambda abstraction case we will use the following observation. By Lemma 4.4(3), for types $X, Y \in \widetilde{\mathbb{B}}$ one has

$$
\begin{aligned}
{[\mathrm{d} \mathcal{V}(-; X), \mathrm{d} \mathcal{N}(-; Y)] } & =[\mathrm{d}(\mathrm{y}[X]), \mathrm{d} \mathcal{N}(-; Y)] \\
& =[\mathrm{Y}[X], \mathrm{d} \mathcal{N}(-; Y)] \\
& \simeq \mathrm{d} \mathcal{N}(-+[X] ; Y)
\end{aligned}
$$

and so one obtains a composite $\mathrm{L}_{X, Y}$ as follows:

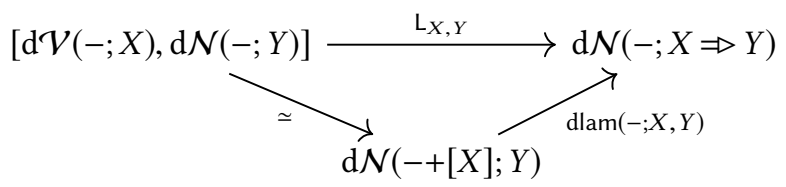

Proposition 4.13 (c.f. [24, Propositions $7 \&$ 8]). For any set of base types $\mathbb{B}$, base type $B \in \mathbb{B}$ and types $A, A_{1}, \ldots, A_{n}$, $X, Y \in \widetilde{\mathbb{B}}(n \in \mathbb{N})$, one obtains the following 1-cells in $\mathrm{gl}(\langle\underline{s}\rangle)$ :

1. $\underline{\operatorname{var}}:=\left(\operatorname{dvar}\left(-; A_{i}\right), \cong, \operatorname{Id}_{s \llbracket A_{i} \rrbracket}\right): v_{A_{i}} \rightarrow \mu_{A_{i}}$.

2. $\underline{\operatorname{proj}}_{k}:=\left(\operatorname{dproj}_{k}\left(-; A_{\bullet}\right), \mathrm{id}, \pi_{k}\right): \mu_{\left(\prod_{i} A_{i}\right)} \rightarrow \mu_{A_{k}}($ for $\bar{k}=1, \ldots, n)$.

3. app $:=\left(\operatorname{dapp}(-; X, Y), \mathrm{id}, \operatorname{eval}_{s \llbracket X \rrbracket, s \llbracket Y \rrbracket}\right)$

$$
: \mu_{X \Rightarrow Y} \times \eta_{X} \rightarrow \mu_{Y} \text {. }
$$

4. $\operatorname{tup}:=\left(\operatorname{dtup}\left(-; A_{\bullet}\right), \cong, \operatorname{Id}_{s \llbracket \prod_{i} A_{i} \rrbracket}\right)$

$$
: \prod_{i=1}^{n} \eta_{A_{i}} \rightarrow \eta_{\left(\prod_{i} A_{i}\right)} \text {. }
$$

5. $\underline{\text { lam }}:=\left(\mathrm{L}_{X, Y}, \cong, \operatorname{Id}_{s \llbracket X \rrbracket \Rightarrow s \llbracket Y \rrbracket}\right):\left(v_{X} \Rightarrow \eta_{Y}\right) \stackrel{\cong}{\rightarrow} \eta_{X \Rightarrow Y}$ for $\mathrm{L}_{X, Y}$ defined in (13).

6. $\underline{\text { inc }}:=\left(\operatorname{inc}(-; B), \cong, \operatorname{Id}_{s \llbracket B \rrbracket}\right): \mu_{B} \stackrel{\cong}{\rightarrow} \eta_{B}$.

In each case, $\cong$ denotes a structural isomorphism or composite of such.

\subsection{Normalisation-by-evaluation}

We are now in a position to prove Theorem 4.1. For any cc-bicategory $\left(\mathcal{X}, \Pi_{n}(-), \Rightarrow\right)$ and set map $s$ as in the preceding section, consider the set map $\tilde{s}: \mathbb{B} \rightarrow \operatorname{gl}(\langle\underline{s}\rangle)$ sending a base type $B$ to the glued object $\mu_{B}$ of (12). This induces an interpretation $\tilde{s} \llbracket-\rrbracket: \mathcal{S}(\mathbb{B}) \rightarrow \operatorname{gl}(\langle\underline{s}\rangle)$. Let us introduce notation for the components of this interpretation. Since the forgetful functor $\pi: \operatorname{gl}(\langle\underline{s}\rangle) \rightarrow \mathcal{X}$ strictly preserves the cc-bicategorical structure (Theorem 4.9), the final component coincides with $s \llbracket-\rrbracket$.

On a type $A \in \widetilde{\mathbb{B}}$, write $\tilde{s} \llbracket A \rrbracket:=\left(G_{A}, \gamma_{A}, s \llbracket A \rrbracket\right)$. On terms, write $\tilde{s} \llbracket \Gamma \vdash t: A \rrbracket:=\left(s^{\prime} \llbracket \Gamma \vdash t: A \rrbracket, \sigma \llbracket \Gamma \vdash t: A \rrbracket, s \llbracket \Gamma \vdash t: A \rrbracket\right)$. For $\Gamma:=\left(x_{i}: A_{i}\right)_{i=1, \ldots, n}$ this is represented pictorially by the following diagram in $\operatorname{Hom}\left(\mathrm{dCon}_{\widetilde{\mathbb{B}}}^{\mathrm{op}}\right.$, Cat $)$ :

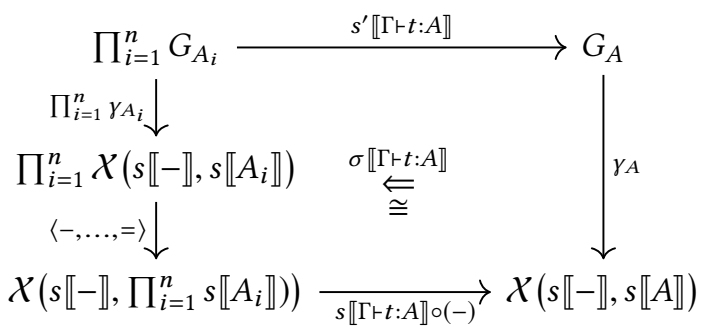

(The left-hand column arises from the fp-structure (11) of the glueing bicategory.) Finally, on rewrites, denote the two components of the 2-cell $\tilde{s} \llbracket \Gamma \vdash \tau: t \Rightarrow t^{\prime}: A \rrbracket$ by

$$
\begin{aligned}
& s^{\prime} \llbracket \Gamma \vdash \tau: t \Rightarrow t^{\prime}: A \rrbracket: s^{\prime} \llbracket \Gamma \vdash t: A \rrbracket \Rightarrow s^{\prime} \llbracket \Gamma \vdash t^{\prime}: A \rrbracket \\
& s \llbracket \Gamma \vdash \tau: t \Rightarrow t^{\prime}: A \rrbracket: s \llbracket \Gamma \vdash t: A \rrbracket \Rightarrow s \llbracket \Gamma \vdash t^{\prime}: A \rrbracket
\end{aligned}
$$

By definition of the glueing bicategory, these 2-cells satisfy the cylinder condition (9).

We now use Proposition 4.13 to define 1-cells unquote ${ }_{A}$ : $\mu_{A} \rightarrow \tilde{s} \llbracket A \rrbracket$ and quote ${ }_{A}: \tilde{s} \llbracket A \rrbracket \rightarrow \eta_{A}$ by induction on types. On a base type $B$ we take

$$
\begin{aligned}
\text { unquote }_{B} & :=\operatorname{Id}_{\mu_{B}}: \mu_{B} \rightarrow \mu_{B}=\tilde{s} \llbracket B \rrbracket \\
\text { quote }_{B} & :=\underline{\text { inc }}^{-1}: \tilde{s} \llbracket B \rrbracket \rightarrow \eta_{B}
\end{aligned}
$$




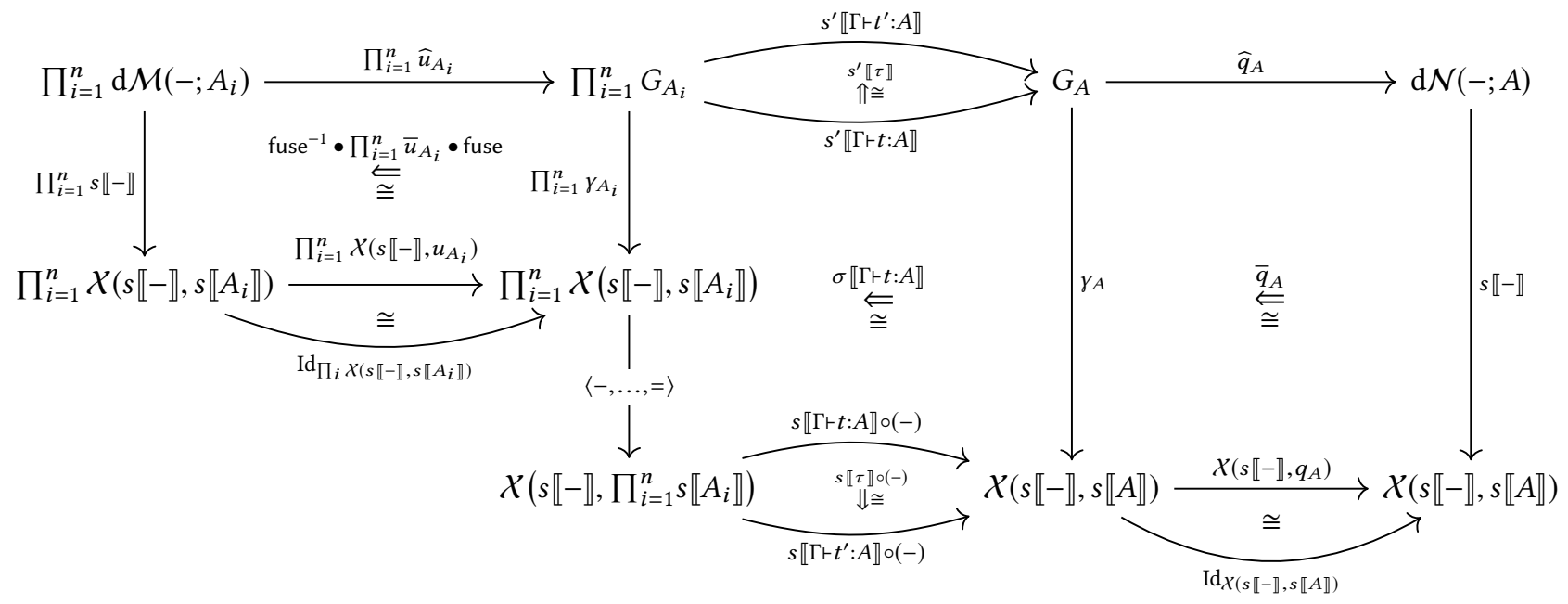

Figure 2. Bicategorical normalisation-by-evaluation (c.f. [24, p. 9]).

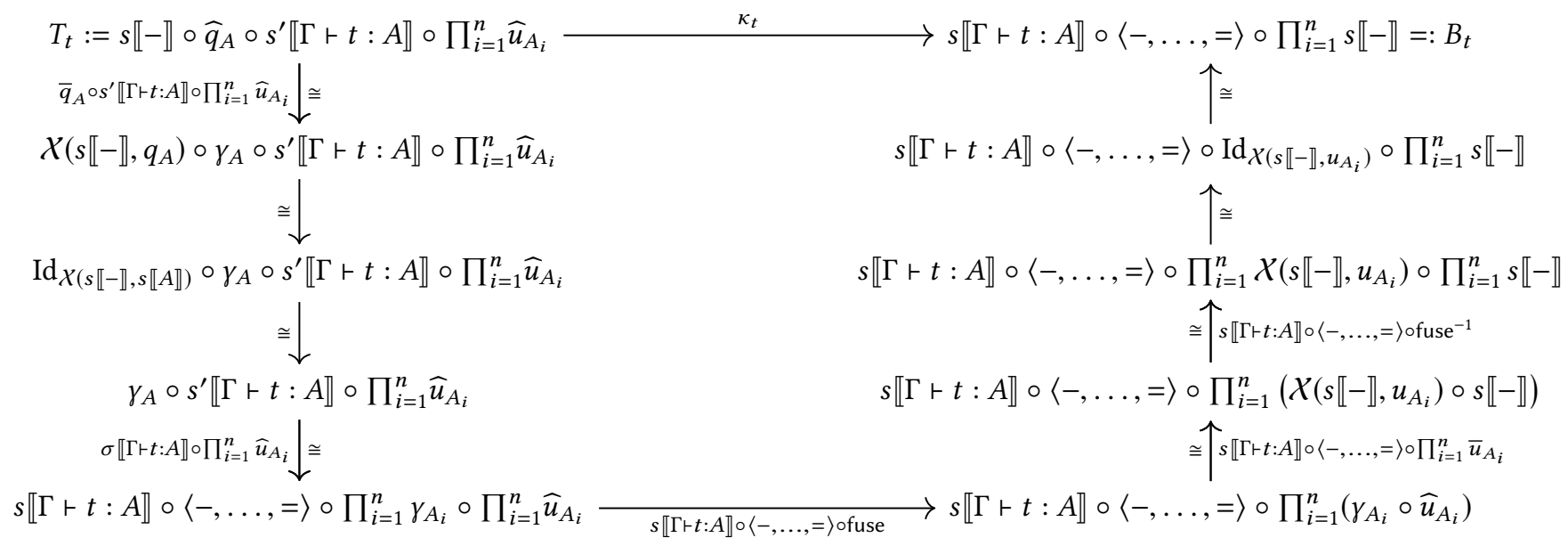

Figure 3. The definition of $\kappa_{t}$.

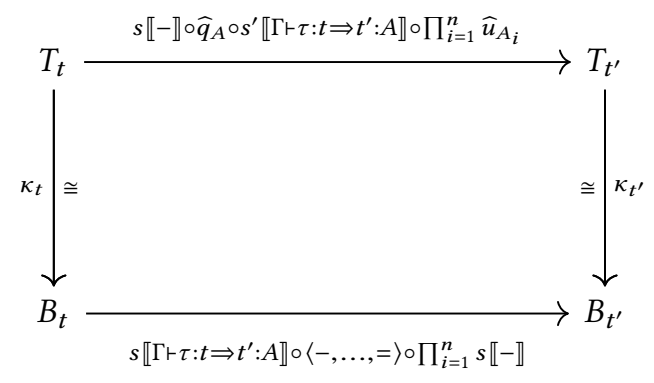

(a) Relating $s \llbracket \Gamma \vdash \tau: t \Rightarrow t^{\prime}: \tau \rrbracket$ and $s^{\prime} \llbracket \Gamma \vdash \tau: t \Rightarrow t^{\prime}: \tau \rrbracket$.

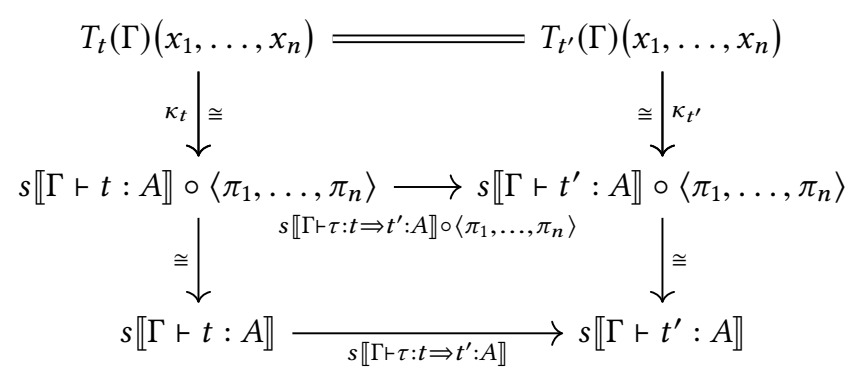

(b) Expressing $s \llbracket \Gamma \vdash \tau: t \Rightarrow t^{\prime}: A \rrbracket$ as a composite independent of $\tau$.

Figure 4. The proof of Proposition 4.15. Here $T_{t}:=s \llbracket-\rrbracket \circ \widehat{q}_{A} \circ s^{\prime} \llbracket \Gamma \vdash t: A \rrbracket \circ \prod_{i=1}^{n} \widehat{u}_{A_{i}}$ is the clockwise route around Figure 2 (along the top) and $B_{t}:=s \llbracket \Gamma \vdash t: A \rrbracket \circ\langle-, \ldots,=\rangle \circ \prod_{i=1}^{n} s \llbracket-\rrbracket$ is the anticlockwise route around Figure 2 (along the bottom). 


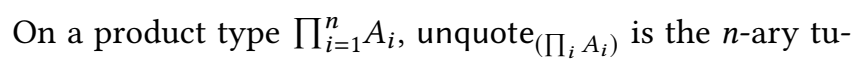
pling of the composite

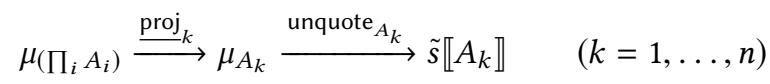

and quote $\left.\prod_{i} A_{i}\right)$ is

$$
\prod_{i=1}^{n} \tilde{s} \llbracket A_{i} \rrbracket \stackrel{\prod_{i=1}^{n} \text { quote }_{A_{i}}}{\longrightarrow} \prod_{i=1}^{n} \eta_{A_{i}} \stackrel{\text { tup }}{\longrightarrow} \eta_{\left(\prod_{i} A_{i}\right)}
$$

For exponential types, unquote $\mathrm{e}_{X \rightarrow Y}$ is the currying of

$\mu_{X \Rightarrow Y} \times \tilde{s} \llbracket X \rrbracket \stackrel{\mu_{X \Rightarrow Y} \times \text { quote }_{X}}{\longrightarrow} \mu_{X \Rightarrow Y} \times \eta_{X} \stackrel{\text { app }}{\longrightarrow} \mu_{Y} \stackrel{\text { unquote }_{Y}}{\longrightarrow} \tilde{s} \llbracket Y \rrbracket$ and quote $_{X \Rightarrow Y}$ is the composite

$$
(\tilde{s} \llbracket X \rrbracket \Rightarrow \tilde{s} \llbracket Y \rrbracket) \rightarrow v_{X} \Rightarrow \eta_{Y} \stackrel{\text { lam }}{\longrightarrow} \eta_{X \Rightarrow Y}
$$

where the first arrow is the currying of

$$
\begin{aligned}
& \text { (quote } \left._{Y} \circ \operatorname{eval}_{\tilde{s} \llbracket X \rrbracket, \tilde{s} \llbracket Y \rrbracket}\right) \\
& \quad \circ\left(\left((\tilde{s} \llbracket X \rrbracket \Rightarrow \tilde{s} \llbracket Y \rrbracket) \times \text { unquote }_{X}\right) \circ((\tilde{s} \llbracket X \rrbracket \Rightarrow \tilde{s} \llbracket Y \rrbracket) \times \underline{\text { var }})\right)
\end{aligned}
$$

Write unquote $_{A}:=\left(\widehat{u}_{A}, \bar{u}_{A}, u_{A}\right)$ and quote ${ }_{A}:=\left(\widehat{q}_{A}, \bar{q}_{A}, q_{A}\right)$, so that $\pi\left(\right.$ unquote $\left._{A}\right)=u_{A}$ and $\pi$ (quote $\left._{A}\right)=q_{A}$. The following is then a straightforward induction using the structural isomorphisms arising from the cartesian closed structure of $\operatorname{gl}(\langle\underline{s}\rangle)$.

Lemma 4.14. For every type $A \in \widetilde{\mathbb{B}}$, there exist natural isomorphisms $u_{A} \cong \operatorname{Id}_{s \llbracket A \rrbracket}$ and $q_{A} \cong \operatorname{Id}_{s \llbracket A \rrbracket}$.

We now argue similarly to our first proof of Theorem 1.1. For $\Gamma:=\left(x_{i}: A_{i}\right)_{i=1, \ldots, n}$ and any rewrite $\Gamma \vdash \tau: t \Rightarrow t^{\prime}: A$ we shall construct a diagram relating $s \llbracket \Gamma \vdash \tau: t \Rightarrow t^{\prime}: A \rrbracket$ and $s^{\prime} \llbracket \Gamma \vdash \tau: t \Rightarrow t^{\prime}: A \rrbracket$ (Figure 4a), then observe this diagram degenerates so that we may express $s \llbracket \Gamma \vdash \tau: t \Rightarrow t^{\prime}: A \rrbracket$ as a composite of 2-cells which do not depend on $\tau$ (Figure $4 b$ ).

We start our construction of Figure 4a by using the 1-cells unquote and quote. Together with the isomorphisms of the preceding lemma and the structural isomorphisms $\operatorname{Id}_{s \llbracket A \rrbracket} \circ$ $f \cong f$, these give rise to the following two diagrams for every type $A \in \widetilde{\mathbb{B}}$ :

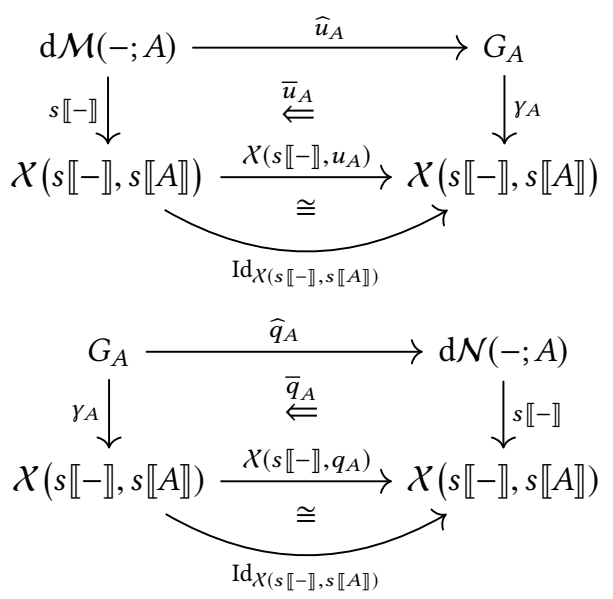

Pasting $\prod_{i=1}^{n} \bar{u}_{A_{i}}$ and $\bar{q}_{A}$ with the 2-cell $\sigma \llbracket \Gamma \vdash t: A \rrbracket$ of (14) and the isomorphism fuse : $\prod_{i=1}^{n} g_{i} \circ \prod_{i=1}^{n} f_{i} \cong \prod_{i=1}^{n}\left(g_{i} \circ f_{i}\right)$ arising from the pseudo-functoriality of $\prod_{i=1}^{n}(-)$, one obtains Figure 2. Now let $\kappa_{t}$ be the (invertible) composite of all the 2-cells in Figure 2 that are independent of $\tau$ : namely, $\bar{q}_{A}, \sigma \llbracket \Gamma \vdash t: A \rrbracket$ fuse $^{-1} \bullet \prod_{i=1}^{n} \bar{u}_{A_{i}} \bullet$ fuse and the structural isomorphisms. This is defined explicitly by Figure 3. Since $s \llbracket \Gamma \vdash \tau: t \Rightarrow t^{\prime}: A \rrbracket$ and $s^{\prime} \llbracket \Gamma \vdash \tau: t \Rightarrow t^{\prime}: A \rrbracket$ are related by the cylinder condition, $\kappa_{t}$ satisfies Figure $4 \mathrm{a}$, in which we write $T_{t}$ for the clockwise composite around Figure 2 (following $s^{\prime} \llbracket \Gamma \vdash t: A \rrbracket$ along the top) and $B_{t}$ for the anticlockwise composite around Figure 2 (following $s \llbracket \Gamma \vdash t: A \rrbracket \circ(-)$ along the bottom).

We now construct Figure $4 \mathrm{~b}$ from Figure $4 \mathrm{a}$. Since $\mathcal{N}(-; A)$ is locally discrete, the top horizontal arrow of Figure $4 \mathrm{a}$ is the identity 2-cell. Hence, recalling that $\Gamma:=\left(x_{i}: A_{i}\right)_{i=1, \ldots, n}$ and since $\left(x_{i}\right)_{A_{i}}^{\Gamma}=x_{i}$, we have

$$
T_{t}(\Gamma)\left(x_{1}, \ldots, x_{n}\right)=T_{t^{\prime}}(\Gamma)\left(x_{1}, \ldots, x_{n}\right)
$$

while, moreover, one calculates that

$$
\begin{aligned}
B_{t}(\Gamma)\left(x_{1}, \ldots, x_{n}\right) & =s \llbracket \Gamma \vdash t: A \rrbracket \circ\left\langle\pi_{1}, \ldots, \pi_{n}\right\rangle \\
B_{t^{\prime}}(\Gamma)\left(x_{1}, \ldots, x_{n}\right) & =s \llbracket \Gamma \vdash t^{\prime}: A \rrbracket \circ\left\langle\pi_{1}, \ldots, \pi_{n}\right\rangle
\end{aligned}
$$

One therefore obtains the top square of Figure $4 \mathrm{~b}$ by instantiating Figure $4 \mathrm{a}$ at $\Gamma$ and evaluating at $\left(x_{1}, \ldots, x_{n}\right)$. The bottom square of Figure $4 \mathrm{~b}$ is obtained by composing with the canonical isomorphism $f \circ\left\langle\pi_{1}, \ldots, \pi_{n}\right\rangle \stackrel{\cong}{\Rightarrow} f(c . f$. diagram (4)). Since each morphism in Figure $4 \mathrm{~b}$ is invertible, it follows that $s \llbracket \Gamma \vdash \tau: t \Rightarrow t^{\prime}: A \rrbracket$ is equal to the clockwise route around this diagram (with the morphisms in the lefthand column inverted). This 2-cell depends only on the context $\Gamma$, the type $A$, and the terms $t$ and $t^{\prime}$, therefore any pair of parallel rewrites must be interpreted by the same 2-cell.

Proposition 4.15. For any set of base types $\mathbb{B}$, cc-bicategory $\left(\mathcal{X}, \Pi_{n}(-), \Rightarrow\right)$, set map $s: \mathbb{B} \rightarrow \mathcal{X}$, and parallel pair of rewrites $\Gamma \vdash \tau: t \Rightarrow t^{\prime}: A$ and $\Gamma \vdash \tau^{\prime}: t \Rightarrow t^{\prime}: A$ in $\Lambda_{\mathrm{ps}}^{\times,} \rightarrow$,

$$
s \llbracket \Gamma \vdash \tau: t \Rightarrow t^{\prime}: A \rrbracket=s \llbracket \Gamma \vdash \tau^{\prime}: t \Rightarrow t^{\prime}: A \rrbracket
$$

We can finally prove Theorem 4.1.

Proof of Theorem 4.1. Instantiate (15) for the inclusion $\iota$ : $\mathbb{B} \hookrightarrow \mathcal{S}(\mathbb{B})$, and apply the local faithfulness of $\iota \llbracket-\rrbracket$ (Proposition 4.2).

Since $\mathcal{S}(\mathbb{B})$ is biequivalent to the free cc-bicategory on $\mathbb{B}$ (Section 4.1.1), this completes the second proof of Theorem 1.1.

\section{Acknowledgments}

We are grateful to André Joyal for suggesting that Power's proof could be adapted to incorporate exponentials.

The second author was supported by a Royal Society University Research Fellow Enhancement Award. 


\section{References}

[1] M. G. Abbott. 2003. Categories of containers. Ph.D. Dissertation. University of Leicester.

[2] A. Abel, T. Coquand, and P. Dybjer. 2007. Normalization by Evaluation for Martin-Löf Type Theory with Typed Equality Judgements. In Proceedings of the 22nd Annual IEEE Symposium on Logic in Computer Science (LICS '07). IEEE Computer Society, Washington, DC, USA, 3-12. https://doi.org/10.1109/LICS.2007.33

[3] D. Ahman and S. Staton. 2013. Normalization by Evaluation and Algebraic Effects. Electronic Notes in Theoretical Computer Science (ENTCS) 298 (2013), 51-69.

[4] M. Alimohamed. 1995. A Characterization of Lambda Definability in Categorical Models of Implicit Polymorphism. Theor. Comput. Sci. 146, 1-2 (July 1995), 5-23. https://doi.org/10.1016/0304-3975(94)00283-O

[5] T. Altenkirch, P. Dybjer, M. Hofmannz, and P. Scott. 2001. Normalization by Evaluation for Typed Lambda Calculus with Coproducts. In Proceedings of the 16th Annual IEEE Symposium on Logic in Computer Science (LICS '01). IEEE Computer Society, Washington, DC, USA, 303-. http://dl.acm.org/citation.cfm?id=871816.871869

[6] T. Altenkirch, M. Hofmann, and T. Streicher. 1995. Categorical reconstruction of a reduction free normalization proof. In Category Theory and Computer Science, 6th International Conference, CTCS '95, Cambridge, UK, August 7-11, 1995, Proceedings, Vol. 953. 182-199.

[7] T. Altenkirch and A. Kaposi. 2016. Normalisation by Evaluation for Dependent Types. In 1st International Conference on Formal Structures for Computation and Deduction (FSCD 2016) (Leibniz International Proceedings in Informatics (LIPIcs)), D. Kesner and B. Pientka (Eds.), Vol. 52. Schloss Dagstuhl-Leibniz-Zentrum fuer Informatik, Dagstuhl, Germany, 6:1-6:16. https://doi.org/10.4230/LIPIcs.FSCD.2016.6

[8] T. Altenkirch and A. Kaposi. 2017. Normalisation by Evaluation for Type Theory, in Type Theory. Logical Methods in Computer Science Volume 13, Issue 4 (Oct. 2017). https://doi.org/10.23638/LMCS-13(4: 1) 2017

[9] S. Awodey. 2010. Category Theory (2nd ed.). Number 52 in Oxford Logic Guides. Oxford University Press.

[10] V. Balat, R. Di Cosmo, and M. Fiore. 2004. Extensional Normalisation and Type-Directed Partial Evaluation for Typed Lambda Calculus with Sums. In Proceedings of the 31st ACM SIGPLAN-SIGACT Symposium on Principles of Programming Languages (Venice, Italy) (POPL '04). Association for Computing Machinery, New York, NY, USA, 64-76. https://doi.org/10.1145/964001.964007

[11] J. Bénabou. 1967. Introduction to bicategories. In Reports of the Midwest Category Seminar. Springer Berlin Heidelberg, Berlin, Heidelberg, 1-77. https://doi.org/10.1007/BFb0074299

[12] U. Berger and H. Schwichtenberg. 1991. An inverse of the evaluation functional for typed lambda-calculus. In Proceedings of the 6th Annual IEEE Symposium on Logic in Computer Science (LICS '91). 203-211. https://doi.org/10.1109/LICS.1991.151645

[13] F. Borceux. 1994. Bicategories and distributors. Encyclopedia of Mathematics and its Applications, Vol. 1. Cambridge University Press, 281-324. https://doi.org/10.1017/CBO9780511525858.009

[14] A. Carboni, G. M. Kelly, R. F. C. Walters, and R. J. Wood. 2008. Cartesian bicategories II. Theory and Applications of Categories 19, 6 (2008), 93 124. http://www.tac.mta.ca/tac/volumes/19/6/19-06abs.html

[15] A. Carboni, S. Lack, and R. F. C. Walters. 1993. Introduction to extensive and distributive categories. Fournal of Pure and Applied Algebra 84, 2 (Feb. 1993), 145-158. https://doi.org/10.1016/0022-4049(93)90035-r

[16] A. Carboni and R. F. C. Walters. 1987. Cartesian bicategories I. fournal of Pure and Applied Algebra 49, 1 (1987), 11-32. https://doi.org/10. 1016/0022-4049(87)90121-6

[17] S. Castellan, P. Clairambault, S. Rideau, and G. Winskel. 2017. Games and Strategies as Event Structures. Logical Methods in Computer Science 13 (2017).
[18] T. Coquand and P. Dybjer. 1997. Intuitionistic Model Constructions and Normalization Proofs. Mathematical. Structures in Comp. Sci. 7, 1 (Feb. 1997), 75-94. https://doi.org/10.1017/S0960129596002150

[19] R. L. Crole. 1994. Categories for Types. Cambridge University Press. https://doi.org/10.1017/CBO9781139172707

[20] D. Čubrić, P. Dybjer, and P. Scott. 1998. Normalization and the Yoneda embedding. Mathematical Structures in Computer Science 8, 2 (1998), 153-192. https://doi.org/10.1017/S0960129597002508

[21] P-E. Dagand and C. McBride. 2013. A Categorical Treatment of Ornaments. In Proceedings of the 2013 28th Annual ACM/IEEE Symposium on Logic in Computer Science (LICS '13). IEEE Computer Society, Washington, DC, USA, 530-539. https://doi.org/10.1109/LICS.2013.60

[22] B. Day. 1970. On closed categories of functors. In Reports of the Midwest Category Seminar IV, S. Mac Lane, H. Applegate, M. Barr, B. Day, E. Dubuc, Phreilambud, A. Pultr, R. Street, M. Tierney, and S. Swierczkowski (Eds.). Springer Berlin Heidelberg, Berlin, Heidelberg, 1-38. https://doi.org/10.1007/BFb0060438

[23] B. Day and R. Street. 1997. Monoidal Bicategories and Hopf Algebroids. Advances in Mathematics 129, 1 (1997), 99-157. https://doi.org/10.1006/ aima.1997.1649

[24] M. Fiore. 2002. Semantic Analysis of Normalisation by Evaluation for Typed Lambda Calculus. In Proceedings of the 4th ACM SIGPLAN International Conference on Principles and Practice of Declarative Programming (Pittsburgh, PA, USA) (PPDP '02). ACM, New York, NY, USA, 26-37. https://doi.org/10.1145/571157.571161

[25] M. Fiore. 2011. Algebraic Foundations for Type Theories. 18th Types for Proofs and Programs workshop. Slides available at https://www.cl. cam.ac.uk/ mpf23/talks/Types2011.pdf.

[26] M. Fiore. 2016. An Algebraic Combinatorial Approach to Opetopic Structure. https://www.mpim-bonn.mpg.de/node/6586. Talk at the Seminar on Higher Structures, Program on Higher Structures in Geometry and Physics, Max Planck Institute for Mathematics, Bonn (Germany).

[27] M. Fiore, N. Gambino, M. Hyland, and G. Winskel. 2007. The cartesian closed bicategory of generalised species of structures. Fournal of the London Mathematical Society 77, 1 (2007), 203-220. https://doi.org/10. 1112/jlms/jdm096

[28] M. Fiore, N. Gambino, M. Hyland, and G. Winskel. 2017. Relative pseudomonads, Kleisli bicategories, and substitution monoidal structures. Selecta Mathematica New Series 24 (2017), 2791-2830. https://doi.org/10.1007/s00029-017-0361-3

[29] M. Fiore and A. Joyal. 2015. Theory of para-toposes. Talk at the Category Theory 2015 Conference. Departamento de Matematica, Universidade de Aveiro (Portugal).

[30] M. Fiore, G. Plotkin, and D. Turi. 1999. Abstract Syntax and Variable Binding. In Proceedings of the 14th Annual IEEE Symposium on Logic in Computer Science (LICS '99). IEEE Computer Society, Washington, DC, USA, 193-. http://dl.acm.org/citation.cfm?id=788021.788948

[31] M. Fiore and P. Saville. 2017. List Objects with Algebraic Structure. In 2nd International Conference on Formal Structures for Computation and Deduction, FSCD 2017, September 3-9, 2017, Oxford, UK. 16:1-16:18. https://doi.org/10.4230/LIPIcs.FSCD.2017.16

[32] M. Fiore and P. Saville. 2019. A type theory for cartesian closed bicategories. In Proceedings of the 34th Annual ACM/IEEE Symposium on Logic in Computer Science (LICS '19). https://doi.org/10.1109/LICS. 2019.8785708

[33] M. Fiore and P. Saville. 2020. Relative Full Completeness for Bicategorical Cartesian Closed Structure. In Foundations of Software Science and Computation Structures, J. Goubault-Larrecq and B. König (Eds.). Springer International Publishing, Cham., 277-298. https: //doi.org/10.1007/978-3-030-45231-5_15

[34] T. Fiore. 2006. Pseudo limits, biadjoints, and pseudo algebras: categorical foundations of conformal field theory. Memoirs of the AMS, Vol. 860 . American Mathematical Society. 
[35] S. Forest and S. Mimram. 2018. Coherence of Gray Categories via Rewriting. In 3rd International Conference on Formal Structures for Computation and Deduction (FSCD '18) (Leibniz International Proceedings in Informatics (LIPIcs)), H. Kirchner (Ed.), Vol. 108. 15:1-15:16. https://doi.org/10.4230/LIPIcs.FSCD.2018.15

[36] N. Gambino and A. Joyal. 2017. On operads, bimodules and analytic functors. Memoirs of the AMS, Vol. 249. American Mathematical Society.

[37] N. Gambino and J. Kock. 2013. Polynomial functors and polynomial monads. Mathematical Proceedings of the Cambridge Philosophical Society 154, 1 (2013), 153-192. https://doi.org/10.1017/S0305004112000394

[38] J.-Y. Girard. 1972. Interprétation fonctionnelle et élimination des coupures de l'arithmétique d'ordre supérieur. Ph.D. Dissertation. Université Paris Diderot - Paris 7.

[39] J.-Y. Girard, P. Taylor, and Y. Lafont. 1989. Proofs and Types. Cambridge University Press, New York, NY, USA.

[40] G.L. Cattani, M. Fiore, and G. Winskel. 1998. A theory of recursive domains with applications to concurrency. In Proceedings of the 13th Annual IEEE Symposium on Logic in Computer Science (LICS '98). IEEE Computer Society, 214-225. https://doi.org/10.1109/LICS.1998.705658

[41] R. Gordon, A. J. Power, and R. Street. 1995. Coherence for tricategories. Memoirs of the AMS, Vol. 558. American Mathematical Society.

[42] J. W. Gray. 1974. Formal Category Theory: Adjointness for 2-Categories. Lecture Notes in Mathematics, Vol. 391. Springer. https://doi.org/10. 1007/BFb0061280

[43] N. Gurski. 2013. Coherence in Three-Dimensional Category Theory. Cambridge University Press. https://doi.org/10.1017/CBO9781139542333

[44] B.P. Hilken. 1996. Towards a proof theory of rewriting: the simply typed $2 \lambda$-calculus. Theoretical Computer Science 170, 1 (1996), 407-444. https://doi.org/10.1016/S0304-3975(96)80713-4

[45] T. Hirschowitz. 2013. Cartesian closed 2-categories and permutation equivalence in higher-order rewriting. Logical Methods in Computer Science 9 (Sept. 2013), 1-22. https://doi.org/10.2168/LMCS-9(3:10)2013

[46] R. Houston. 2007. Linear Logic without Units. Ph.D. Dissertation. University of Manchester.

[47] A. Joyal and R. Street. 1993. Braided tensor categories. Advances in Mathematics 102, 1 (11 1993), 20-78. https://doi.org/10.1006/aima. 1993.1055

[48] S. Lack. 2010. A 2-Categories Companion. Springer New York, New York, NY, 105-191. https://doi.org/10.1007/978-1-4419-1524-5_4

[49] S. Lack, R. F. C. Walters, and R. J. Wood. 2010. Bicategories of spans as cartesian bicategories. Theory and Applications of Categories 24, 1 (2010), 1-24. http://www.tac.mta.ca/tac/volumes/24/1/24-01.pdf

[50] J. Lambek and P. J. Scott. 1986. Introduction to Higher Order Categorical Logic. Cambridge University Press, New York, NY, USA.

[51] T. Leinster. 1998. Basic Bicategories. (May 1998). Available at https: //arxiv.org/abs/math/9810017.

[52] T. Leinster. 2004. Higher operads, higher categories. Number 298 in London Mathematical Society Lecture Note Series. Cambridge University Press.

[53] Q. M. Ma and J. C. Reynolds. 1992. Types, abstraction, and parametric polymorphism, part 2. In Mathematical Foundations of Programming Semantics, S. Brookes, M. Main, A. Melton, M. Mislove, and D. Schmidt (Eds.). Springer Berlin Heidelberg, Berlin, Heidelberg, 1-40.

[54] S. Mac Lane. 1963. Natural associativity and commutativity. Rice University Studies (1963). https://hdl.handle.net/1911/62865

[55] S. Mac Lane. 1998. Categories for the Working Mathematician (second ed.). Graduate Texts in Mathematics, Vol. 5. Springer-Verlag New York. https://doi.org/10.1007/978-1-4757-4721-8

[56] S. Mac Lane and R. Paré. 1985. Coherence for bicategories and indexed categories. Journal of Pure and Applied Algebra 37 (1985), 59-80. https: //doi.org/10.1016/0022-4049(85)90087-8

[57] M. Makkai. 1996. Avoiding the axiom of choice in general category theory. Journal of Pure and Applied Algebra 108, 2 (1996), 109-173. https://doi.org/10.1016/0022-4049(95)00029-1

[58] Federico Olimpieri. 2020. Intersection Type Distributors. arXiv (2020). arXiv:2002.01287v2 [cs.LO]

[59] J. Ouaknine. 1997. A two-dimensional extension of Lambek's categorical proof theory. Master's thesis. McGill University. http://digitool.library.mcgill.ca/R/?func=dbin-jump-full\&object_ id $=20277 \&$ local_base $=$ GEN01-MCG02

[60] H. Paquet. 2020. Probabilistic concurrent game semantics. Ph.D. Dissertation. University of Cambridge.

[61] A. M. Pitts. 1987. An elementary calculus of approximations (extended abstract). (1987). Unpublished manuscript, University of Sussex, December 1987.

[62] A. M. Pitts. 2000. Categorical Logic. In Handbook of Logic in Computer Science. Oxford University Press, Oxford, UK, Chapter 2, 39-123. http: //dl.acm.org/citation.cfm?id=373919.373928

[63] A. J. Power. 1989. Coherence for Bicategories with Finite Bilimits I. In Categories in Computer Science and Logic: Proceedings of the AMSIMS-SIAM Joint Summer Research Conference Held June 14-20, 1987 with Support from the National Science Foundation, J. W. Gray and A. Scedrov (Eds.). Vol. 92. American Mathematical Society, 341-349.

[64] A. J. Power. 1989. A general coherence result. Journal of Pure and Applied Algebra 57, 2 (1989), 165-173. https://doi.org/10.1016/00224049(89)90113-8

[65] E. Ritter and V. de Paiva. 1997. On explicit substitutions and names (extended abstract). In Automata, Languages and Programming, P. Degano, R. Gorrieri, and A. Marchetti-Spaccamela (Eds.). Springer Berlin Heidelberg, Berlin, Heidelberg, 248-258. https://doi.org/10.1007/3-54063165-8_182

[66] P. Saville. forthcoming. Cartesian closed bicategories: type theory and coherence. Ph.D. Dissertation. University of Cambridge.

[67] R. A. G. Seely. 1987. Modelling computations: a 2-categorical framework. In Proceedings of the 2nd Annual IEEE Symp. on Logic in Computer Science (LICS '87) (Ithaca, NY, USA), D. Gries (Ed.). IEEE Computer Society Press, 65-71.

[68] R. Statman. 2014. Near semi-rings and lambda calculus. In Rewriting and Typed Lambda Calculi: foint International Conference, RTA-TLCA 2014, Held as Part of the Vienna Summer of Logic, VSL 2014, Vienna, Austria, fuly 14-17, 2014. Proceedings, G. Dowek (Ed.). Springer International Publishing, Cham., 410-424. https://doi.org/10.1007/978-3319-08918-8_28

[69] S. Staton. 2013. An algebraic presentation of predicate logic. In Foundations of Software Science and Computation Structures, F. Pfenning (Ed.). Springer Berlin Heidelberg, Berlin, Heidelberg, 401-417. https://doi.org/10.1007/978-3-642-37075-5_26

[70] R. Street. 1972. The formal theory of monads. Journal of Pure and Applied Algebra 2, 2 (1972), 149-168. https://doi.org/10.1016/00224049(72)90019-9

[71] R. Street. 1980. Fibrations in bicategories. Cahiers de Topologie et Géométrie Différentielle Catégoriques 21, 2 (1980), 111-160. http:// eudml.org/doc/91227

[72] R. Street. 1995. Categorical Structures. In Handbook of Algebra, M. Hazewinkel (Ed.). Vol. 1. Elsevier, Chapter 15, 529-577. http: //maths.mq.edu.au/ street/45.pdf

[73] W. Tait. 1967. Intensional Interpretations of Functionals of Finite Type I. The fournal of Symbolic Logic 32, 2 (1967), 198-212. http: //www.jstor.org/stable/2271658

[74] T. Uustalu. 2014. Coherence for Skew-Monoidal Categories. Electronic Proceedings in Theoretical Computer Science 153 (June 2014). https: //doi.org/10.4204/EPTCS.153.5 